\title{
Pacific
}

Journal of

Mathematics

\section{GENERALIZED TWISTED SECTORS OF ORBIFOLDS}

CARla Farsi ANd Christopher SEATON 


\title{
GENERALIZED TWISTED SECTORS OF ORBIFOLDS
}

\author{
CARla FARSi AND CHRISTOPHer SEATON
}

\begin{abstract}
For a finitely generated discrete group $\Gamma$, the $\Gamma$-sectors of an orbifold $Q$ are a disjoint union of orbifolds corresponding to homomorphisms from $\Gamma$ into a groupoid presenting $Q$. Here, we show that the inertia orbifold and $k$-multisectors are special cases of the $\Gamma$-sectors, and that the $\Gamma$-sectors are orbifold covers of Leida's fixed-point sectors. In the case of a global quotient, we show that the $\Gamma$-sectors correspond to orbifolds considered by other authors for global quotient orbifolds, as well as their direct generalization to the case of an orbifold given by a quotient by a Lie group. Furthermore, we develop a model for the $\Gamma$-sectors corresponding to a generalized loop space.
\end{abstract}

\section{Introduction}

In [FS 2010], we introduced the $\Gamma$-sectors of an orbifold in order to determine a complete obstruction to the existence of a nonvanishing vector field. The definitions of these sectors were heavily motivated by several existing constructions for orbifolds by Kawasaki [1978; 1979; 1981], Chen and Ruan [2004; Ruan 2002], Bryan and Fulman [1998], and Tamanoi [2001; 2003].

The goal of this paper is to show explicitly how the $\Gamma$-sectors generalize these constructions. In particular, we show that the inertia orbifold corresponds to the $\mathbb{Z}$-sectors and that the $k$-multisectors correspond to the $\mathbb{F}_{k}$-sectors, where $\mathbb{F}_{k}$ is the free group with $k$ generators. The orbifolds whose Euler characteristics were considered by Bryan, Fulman and Tamanoi for global quotients correspond to the $\mathbb{Z}^{k}$-sectors and $\Gamma$-sectors, respectively, in the case where $Q$ can be expressed as a global quotient, that is, a quotient of a manifold by a finite group. We also show that the fixed-point sectors introduced by Leida [2005] are orbifold-covered by the $\Gamma$-sectors for an appropriate choice of $\Gamma$.

Lupercio and Uribe [2002] (see also [de Fernex et al. 2006]) demonstrated that the inertia orbifold naturally appears when one considers the loop space of an orbifold. Here, we show that the same holds for the $\Gamma$-sectors; in particular, they

MSC2000: primary 22A22, 57S15; secondary 57S17, 58H05.

Keywords: orbifold, orbifold groupoid, twisted sector, loop groupoid.

Seaton was partially supported by a Rhodes College Faculty Development Endowment Grant. 
appear when we consider smooth maps $M_{\Gamma} \rightarrow Q$, where $M_{\Gamma}$ is a smooth manifold with fundamental group $\Gamma$. This generalizes results of [Tamanoi 2003], stated for global quotients in the context of orbifold bundles.

In the case where an orbifold $Q$ is presented by a quotient $M / G$, where $M$ is a manifold and $G$ is a Lie group acting locally freely, that is, properly with discrete stabilizers, there is a very natural extension of the orbifold definitions of Bryan, Fulman and Tamanoi; see Definition 2.1. We show that this again coincides with the $\Gamma$-sectors. Note, however, that such a presentation of the $\Gamma$-sectors leads to a different indexing of the sectors. In the case of a global quotient, the $\Gamma$-sectors are naturally indexed by $G$-conjugacy classes $(\phi) \sim$ of homomorphisms $\phi: \Gamma \rightarrow G$ whose images fix a nonempty subset of $M$; we denote by $t_{M ; G}^{\Gamma}$ the set of conjugacy classes of such homomorphisms; see Section 2A. On the other hand, if $\mathscr{G}$ is an

orbifold groupoid presenting $Q$, the sectors are indexed by elements of $T_{Q}^{\Gamma}$, the set of $\approx$-classes of elements of $\operatorname{Hom}(\Gamma, \mathscr{G})$ or equivalently connected components of $|\mathscr{G} \ltimes \operatorname{Hom}(\Gamma, \mathscr{G})|$; see [FS 2010, Subsection 2.2] or Section 2B below. The discrepancy arises because the fixed-point set of a homomorphism $\phi: \Gamma \rightarrow G$ need not be connected. Hence the $\Gamma$-sector corresponding to $(\phi) \sim \in t_{M ; G}^{\Gamma}$ may correspond to the disjoint union of several $\Gamma$-sectors, each corresponding to one element of $T_{Q}^{\Gamma}$; see Example 3.2.

In [FS 2010], we required that our local groups act with a fixed-point set of codimension 2; however, we noted that the construction of the $\Gamma$-sectors did not need this property, and we do not retain this requirement here. While our primary interest is the case of orbifold groupoids, many of these constructions and results generalize directly to the case of orbispaces [Chen 2006], as noted below.

\section{Two definitions of the $\Gamma$-sectors for quotient orbifolds}

In [FS 2010], we constructed the $\Gamma$-sectors of a general orbifold in terms of the orbifold structure given by an orbifold groupoid $\mathscr{G}$, that is, a proper, étale Lie groupoid. For background on orbifolds from this perspective, see [Adem et al. 2007] and also [Moerdijk and Mrčun 2003; Moerdijk 2002]. Section 2A primarily concerns orbifolds presented as the quotient of a manifold by a Lie group. We construct the $\Gamma$-sectors directly from such a presentation. This construction was introduced by Tamanoi [2001; 2003] for the case where $G$ is finite; the definitions are unchanged for general $G$. Section 2B reviews the key points of the construction in [FS 2010] and gives other interpretations. To distinguish from the construction using a general orbifold groupoid, we use slightly different notation for the $\Gamma$-sectors of a quotient orbifold; these definitions will be compared in Section 3.

2A. $\Gamma$-sectors of a quotient presentation. Let $Q$ be an $n$-dimensional quotient orbifold. By this, we mean that $Q$ is presented by $G \ltimes M$, where $M$ is a smooth 
manifold, $G$ is a Lie group acting smoothly on $M$, and $G \ltimes M$ is Morita equivalent to an orbifold groupoid, that is, a proper étale Lie groupoid. In [Adem and Ruan 2003, page 536] and [Adem et al. 2007, page 57] (and see also [Kawasaki 1978, page 76]), it is noted that this is the case whenever these conditions are satisfied:

(i) The isotropy group $G_{x}$ for each $x \in M$ is finite.

(ii) There is a smooth slice $S_{x}$ at each $x \in M$.

(iii) For each $x, y \in M$ with $y \notin G x$, there are slices $S_{x}$ and $S_{y}$ such that

$$
G S_{x} \cap G S_{y}=\varnothing .
$$

In particular, (ii) and (iii) are automatically satisfied if $G$ is compact. The following special cases are worth noting; occasionally, we will restrict our attention to one of these.

- If $G$ is a finite group, then $Q$ is a global quotient orbifold.

- If $G$ is a discrete group acting properly discontinuously, then $Q$ is a good orbifold [Thurston 1978, Definition 13.2.3; Boileau et al. 2003, page 20].

We use $M / G$ to denote the quotient as a topological space with quotient map $\sigma: M \rightarrow M / G$; the orbifold (that is, the Morita equivalence class of the groupoid $G \ltimes M)$ will generally be denoted $Q$. Henriques and Metzler [2004] addressed the question of whether every orbifold can be expressed as a quotient; this question remains unresolved in general.

In the case of a good orbifold (including the case of a global quotient), the groupoid $G \ltimes M$ is an orbifold groupoid. On the other hand, if $G$ is a Lie group of positive dimension, then $G \ltimes M$ is not étale, though it is Morita equivalent to an orbifold groupoid. In general, $G \ltimes M$ as well as any Morita equivalent groupoid will always be a proper foliation groupoid; see [Adem et al. 2007, pages 18 and 21] and [Crainic and Moerdijk 2001] for more details.

Let $\Gamma$ be a finitely generated discrete group - although many of our constructions make sense for arbitrary $\Gamma$, we are only interested in this case. If $\phi$ and $\psi$ are homomorphisms from $\Gamma$ to $G$, we say $\phi \sim \psi$ if they are pointwise conjugate, that is, if there is a $g \in G$ such that $g \phi(\gamma) g^{-1}=\psi(\gamma)$ for each $\gamma \in \Gamma$. We let $(\phi)$ denote the conjugacy class of $\phi$ (or sometimes $(\phi) \sim$ to distinguish from equivalence classes via other relations), and let $t_{M ; G}^{\Gamma}$ denote the set of conjugacy classes of homomorphisms $\phi$ whose images have nonempty fixed-point sets in $M$. We let $M^{\langle\phi\rangle}$ denote the fixed-point set of the image of $\phi$ in $G$, and $C_{G}(\phi)$ the centralizer of the image of $\phi$ in $G$.

Definition 2.1. Let $\phi: \Gamma \rightarrow G$ be a homomorphism with $M^{\langle\phi\rangle} \neq \varnothing$. Then the $\Gamma$-sector of $G \ltimes M$ associated to $(\phi)$ is the orbifold with presentation

$$
(M ; G)_{(\phi)}:=C_{G}(\phi) \ltimes M^{\langle\phi\rangle} .
$$


We let $(M ; G)_{\Gamma}$ denote the disjoint union of the $\Gamma$-sectors:

$$
(M ; G)_{\Gamma}:=\coprod_{(\phi) \in t_{M ; G}^{\Gamma}}(M ; G)_{(\phi)} .
$$

If $G$ is finite, it is obvious that each $(M ; G)_{(\phi)}$ is an orbifold groupoid (that is, a proper étale Lie groupoid). In Corollary 3.3, we will see this is generally the case.

If $x \in M^{\langle\phi\rangle} \subseteq M$, we will sometimes use the notation $(x, \phi)$ to distinguish between $(x, \phi) \in M^{\langle\phi\rangle}$ and $(x, 1) \in M^{\langle 1\rangle}=M$. Hence, we use $C_{G}(\phi)(x, \phi)$ to denote the corresponding point in the orbit space of $(M ; G)_{(\phi)}$.

The following lemma, whose proof is standard, ensures that the definition of $(M ; G)_{(\phi)}$ does not depend on the choice of the representative of the class $(\phi)$.

Lemma 2.2. Let $G$ be a group acting on the smooth manifold $M$ such that $G \ltimes M$ presents a smooth orbifold, and let $\Gamma$ be a finitely generated discrete group. If $\phi, \psi: \Gamma \rightarrow G$ are conjugate homomorphisms with $\psi=g \phi g^{-1}$ for $g \in G$, then the map

$$
L_{g}: M^{\langle\phi\rangle} \rightarrow M^{\langle\psi\rangle}, \quad(x, \phi) \mapsto\left(g x, g \phi g^{-1}\right)=(g x, \psi)
$$

is a $C_{G}(\phi)-C_{G}(\psi)$-equivariant diffeomorphism inducing a groupoid isomorphism between $(M ; G)_{(\phi)}$ and $(M ; G)_{(\psi)}$. Also, $\left.\sigma\right|_{M^{\langle\phi\rangle}}=\left.\sigma\right|_{M^{\langle\psi\rangle}} \circ L_{g}$.

In particular, $G$ acts on the set $\bigsqcup_{\phi \in \operatorname{Hom}(\Gamma, G)}\left(M^{\langle\phi\rangle}, \phi\right)$ by defining $g(x, \phi)=$ $\left(g x, g \phi g^{-1}\right)$. The next lemma introduces a different presentation for $(M ; G)_{\Gamma}$.

Lemma 2.3. Suppose $G \ltimes M$ presents a quotient orbifold, and let $\Gamma$ be a finitely generated discrete group. There is a strong equivalence

$$
G \ltimes \coprod_{\psi \in \operatorname{Hom}(\Gamma, G)}\left(M^{\langle\psi\rangle}, \psi\right) \rightarrow(M ; G)_{\Gamma} .
$$

Hence, $G \ltimes \coprod_{\psi \in \operatorname{Hom}(\Gamma, G)}\left(M^{\langle\psi\rangle}, \psi\right)$ and $(M ; G)_{\Gamma}$ are Morita equivalent.

By strong equivalence, we mean an equivalence of groupoids such that the map on objects is a surjective submersion [Adem et al. 2007, page 20]. Neither of the groupoids in question need be orbifold groupoids; we will see in Section 3 that they are both Morita equivalent to orbifold groupoids.

Proof. Pick $\phi \in \operatorname{Hom}(\Gamma, G)$ with $M^{\langle\phi\rangle} \neq \varnothing$. Here, we denote points in $M^{\langle\phi\rangle}$ simply as $x$ to distinguish from points in $\coprod_{\psi \in(\phi)}\left(M^{\langle\psi\rangle}, \psi\right)$. For each $\psi \in(\phi)$, pick a $g_{\psi} \in G$ such that $g_{\psi} \psi g_{\psi}^{-1}=\phi$. We require that $g_{\phi}=1$. Define the map

$$
\Psi_{0}^{\phi}: \coprod_{\psi \in(\phi)}\left(M^{\langle\psi\rangle}, \psi\right) \rightarrow M^{\langle\phi\rangle}, \quad(x, \psi) \mapsto g_{\psi} x
$$


Similarly, since $G$ acts on $\coprod_{\psi \in(\phi)}\left(M^{\langle\psi\rangle}, \psi\right)$, define $\Psi_{1}^{\phi}: G \times \coprod_{\psi \in(\phi)}\left(M^{\langle\psi\rangle}, \psi\right) \rightarrow C_{G}(\phi) \times M^{\langle\phi\rangle}, \quad(g,(x, \psi)) \mapsto\left(g_{\left(g \psi g^{-1}\right)} g g_{\psi}^{-1}, g_{\psi} x\right)$. It is easy to check that $\Psi_{0}^{\phi}$ and $\Psi_{1}^{\phi}$ are smooth, and that they form the maps on objects and arrows, respectively, of a groupoid homomorphism

$$
\Psi^{\phi}: G \ltimes \coprod_{\psi \in(\phi)}\left(M^{\langle\psi\rangle}, \psi\right) \rightarrow C_{G}(\phi) \ltimes M^{\langle\phi\rangle} .
$$

Since $\Psi_{0}^{\phi}$ is a disjoint union of diffeomorphisms, $\Psi_{0}^{\phi}$ is a surjective submersion. It remains to show that

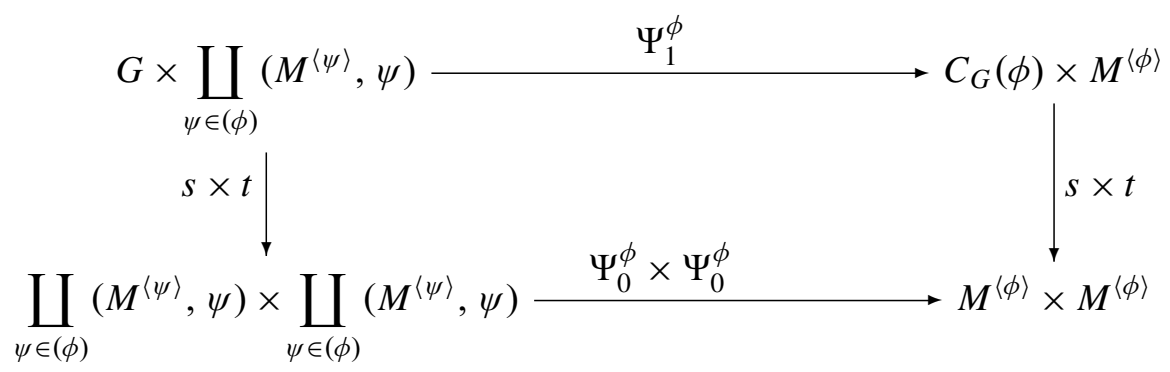

is a fibered product of manifolds. This follows from the fact that the map

$$
\begin{aligned}
\Phi^{\phi}: G \times \coprod_{\psi \in(\phi)}\left(M^{\langle\psi\rangle}, \psi\right) & \rightarrow \coprod_{\psi \in(\phi)}\left(M^{\langle\psi\rangle}, \psi\right) \times \coprod_{\psi \in(\phi)}\left(M^{\langle\psi\rangle}, \psi\right) \times\left(C_{G}(\phi) \times M^{\langle\phi\rangle}\right), \\
(h,(w, \psi)) & \mapsto\left((w, \psi),\left(h w, h \psi h^{-1}\right),\left(g_{\left(h \psi h^{-1}\right)} h g_{\psi}^{-1}, g_{\psi} w\right)\right)
\end{aligned}
$$

is a diffeomorphism onto the fibered product, which is easy to verify.

With this, we need only note that $G \ltimes \coprod_{\phi \in \operatorname{Hom}(\Gamma, G)}\left(M^{\langle\phi\rangle}, \phi\right)$ admits a decomposition into disjoint groupoids

$$
G \ltimes \coprod_{\phi \in \operatorname{Hom}(\Gamma, G)}\left(M^{\langle\phi\rangle}, \phi\right)=\coprod_{(\phi) \in t_{M ; G}^{\Gamma}} G \ltimes \coprod_{\psi \in(\phi)}\left(M^{\langle\psi\rangle}, \psi\right),
$$

and each $\Psi^{\phi}$ maps one of these groupoids into $(M ; G)_{(\phi)}$. Hence,

$$
\Psi:=\coprod_{(\phi) \in t_{M ; G}^{\Gamma}} \Psi^{\phi}: G \ltimes \coprod_{\phi \in \operatorname{Hom}(\Gamma, G)}\left(M^{\langle\phi\rangle}, \phi\right) \rightarrow(M ; G)_{\Gamma}
$$

is clearly surjective, and therefore is a strong equivalence.

The maps $\Psi^{\phi}$ depend on the choice of the $g_{\psi} \in G$; however, it is easy to see that the induced map on orbit spaces does not. 
Fix a homomorphism $\phi: \Gamma \rightarrow G$. Then the injection $M^{\langle\phi\rangle} \hookrightarrow M$ induces a map

$$
\pi_{(\phi)}: M^{\langle\phi\rangle} / C_{G}(\phi) \rightarrow M / G, \quad C_{G}(\phi)(x, \phi) \mapsto G x .
$$

If $g \phi g^{-1}=\psi$ and $(x, \phi) \in M^{\langle\phi\rangle}$, then the $G$-orbit of $x$ in $M$ coincides with that of the corresponding point $g(x, \phi) \in M^{\langle\psi\rangle}$. Therefore, this map does not depend on the choice of representative from the conjugacy class $(\phi)$. If $(x, \phi) \in M^{\langle\phi\rangle}$, then $\sigma(x)=\pi_{(\phi)}\left(C_{G}(\phi)(x, \phi)\right)$. In particular, $\pi_{(\phi)}\left(M^{\langle\phi\rangle}\right)=\sigma\left(M^{\langle\phi\rangle}\right)$.

Finally, the map $M / C_{G}(\phi) \rightarrow M / G, C_{G}(\phi)(x, \phi) \mapsto G x$ is an orbifold cover by definition [Adem et al. 2007, Definition 2.16]. The map $\pi_{(\phi)}$ is the restriction of this orbifold cover to $M^{\langle\phi\rangle} / C_{G}(\phi)$.

2B. $\Gamma$-sectors for a general presentation. We now review the construction of the $\Gamma$-sectors for a general orbifold $Q$. We state it in general for an arbitrary orbifold groupoid $\mathscr{G}$. Throughout, we let the groupoid $\mathscr{G}$ have space of objects $G_{0}$ and space of arrows $G_{1}$. We also let $\sigma: G_{0} \rightarrow|\mathscr{G}|$ denote the quotient map.

If $\Gamma$ and $\mathscr{G}$ are groupoids (with no additional hypotheses), then let $\mathscr{S}_{\mathscr{G}}^{\Gamma}$ denote the set of groupoid homomorphisms $\phi: \Gamma \rightarrow \mathscr{G}$ such that the map on objects is constant. Then $\mathscr{G}$ acts on $\mathscr{S}_{\mathscr{G}}^{\Gamma}$ by conjugation; if $\phi_{0}(z)=x$ for each $z \in \Gamma_{0}$, then for each $g \in G_{1}$ with $s(g)=x$, we let $(g \cdot \phi): \Gamma \rightarrow \mathscr{G}$ have constant map on objects with value $t(g)$ and map on arrows $(g \cdot \phi)_{1}(\gamma)=g \phi_{1}(\gamma) g^{-1}$ for each $\gamma \in \Gamma_{1}$.

If $\Gamma$ is a group (treated as a groupoid with one unit), then every homomorphism $\Gamma \rightarrow \mathscr{G}$ is constant on objects and corresponds to choice of $x \in G_{0}$ and group homomorphism $\phi_{x}: \Gamma \rightarrow G_{x}$, where $G_{x}$ denotes the isotropy group of $x$. We also use $\phi_{x}$ to denote the corresponding groupoid homomorphism.

If $\varphi$ is a topological groupoid presenting an orbispace $X$ [Chen 2006; Henriques and Gepner 2007], then each point $x \in G_{0}$ is contained in an open, connected, locally connected $U \subseteq G_{0}$ such that $G_{\left.\right|_{U}}$ is isomorphic to $G_{U} \ltimes U$, where $G_{U}$ is a topological group acting continuously on $U$. We give $\mathscr{S}_{\mathscr{G}}^{\Gamma}$ the weak topology induced by the maps $\beta \varphi_{g}: \phi_{x} \mapsto x \in G_{0}$ and the evaluation $\epsilon_{\gamma}: \phi_{x} \mapsto \phi_{x}(\gamma) \in G_{1}$ for each $\gamma \in \Gamma$. It is easy to check that the $\mathscr{G}_{\mathcal{G}}$-action on $\mathscr{S}_{\mathscr{G}}^{\Gamma}$ is continuous.

Definition 2.4. Let $\varphi$ be a topological groupoid representing an orbispace $X$, and let $\Gamma$ be a finitely generated discrete group. The $\Gamma$-sector groupoid $\mathscr{G}$ of $\mathscr{G}$ is the translation groupoid $\varphi_{\mathscr{G}} \ltimes \mathscr{Y}_{\mathscr{G}}^{\Gamma}$.

For each $\phi_{x} \in \mathscr{S}_{\mathscr{G}}^{\Gamma}$, choosing $x \in U \subseteq \mathscr{Y}_{\mathscr{G}}^{\Gamma}$ as above induces an isomorphism of topological groupoids between $C_{G_{U}}\left(\phi_{x}\right) \ltimes U^{\left\langle\phi_{x}\right\rangle}$ and the restriction of $\varphi^{\Gamma}$ to the connected component of $\beta_{\Gamma}^{-1}(U)$ containing $\phi_{x}$. It follows that the $\Gamma$-sector groupoid represents an orbispace $\left|\varphi^{\Gamma}\right|$. As a set, we have

$$
\left|\mathscr{G}^{\Gamma}\right|=\left\{\left(p,\left(\phi_{x}\right)_{G_{x}}\right): p=\mathscr{G} x \in\left|\mathscr{G}_{\mid}\right|, \phi_{x} \in \operatorname{Hom}\left(\Gamma, G_{x}\right)\right\},
$$

where $\left(\phi_{x}\right)_{G_{x}}$ denotes the conjugacy class of the homomorphism $\phi_{x}$ in $G_{x}$. 
Now assume that $\mathscr{G}$ is an orbifold groupoid presenting the orbifold $Q$, and $\Gamma$ is a finitely generated discrete group. Then if $\phi_{x}, \psi_{y} \in \mathscr{S}_{\mathscr{G}}^{\Gamma}$, a natural transformation from $\phi_{x}$ to $\psi_{y}$ is simply a choice of an arrow $g \in G_{1}$ such that $s(g)=x, t(g)=y$, and $\psi_{x}(\gamma) g=g \phi_{y}(\gamma)$ for each $\gamma \in \Gamma$. Moreover, if $\epsilon: \mathscr{K} \rightarrow \Gamma$ is an equivalence, then $\epsilon$ is locally invertible, and $\phi_{x} \circ \epsilon^{-1}$ is equivalent to $\phi_{x}$ [Adem et al. 2007, Example 2.42]. It follows that the orbits of points in $\mathscr{S}_{\mathscr{G}}^{\Gamma}$ via the $\mathscr{G}_{\text {-action }}$ correspond exactly to groupoid morphisms from $\Gamma$ to $\mathscr{G}$.

For each point $p \in Q$ corresponding to the orbit of $x \in G_{0}$, there is a linear orbifold chart $\left\{V_{x}, G_{x}, \pi_{x}\right\}$ for $Q$ at $x$. That is, $V_{x} \subseteq G_{0}$ is diffeomorphic to $\mathbb{R}^{n}$ with $x$ corresponding to the origin, $G_{x}$ acts linearly on $V_{x}$, and there is a groupoid isomorphism between $G_{V_{x}}$ and $G_{x} \ltimes V_{x}$. We let $\xi_{x}:(s, t)^{-1}\left(V_{x} \times V_{x}\right) \rightarrow G_{x}$ denote the identification given by this isomorphism, and $\xi_{x}^{y}=\left.\left(\xi_{x}\right)\right|_{G_{y}}: G_{y} \rightarrow G_{x}$ the injective homomorphism given by restriction to $G_{y}$ for each $y \in V_{x}$.

In this case, $\mathscr{Y}_{\varphi}^{\Gamma}$ is a smooth manifold (with connected components of different

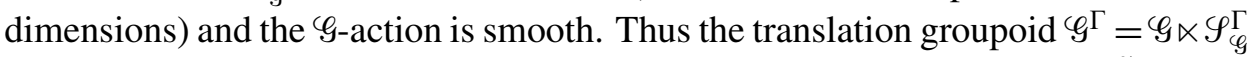
is an orbifold groupoid, defining an orbifold structure for the $\Gamma$-sectors $\tilde{Q}_{\Gamma}$ of $Q$. For each $\phi_{x} \in \mathscr{Y}_{\mathscr{G}}^{\Gamma}$, there is a diffeomorphism $\kappa_{\phi_{x}}$ of $V_{x}^{\left\langle\phi_{x}\right\rangle}$ onto a neighborhood of $\phi_{x}$ in $\mathscr{S}_{\mathscr{G}}^{\Gamma}$ forming a manifold chart. Identifying $V_{x}^{\left\langle\phi_{x}\right\rangle}$ with its image via $\kappa_{\phi_{x}}$, we see that $\left\{V_{x}^{\left\langle\phi_{x}\right\rangle}, C_{G_{x}}\left(\phi_{x}\right), \pi_{x}^{\phi_{x}}\right\}$ forms a linear orbifold chart for $\tilde{Q}_{\Gamma}$ at $\phi_{x}$.

Within a linear chart $\left\{V_{x}, G_{x}, \pi_{x}\right\}$ at $x$ with $y \in V_{x}$, we say $\phi_{x}$ locally covers $\psi_{y}$ (and write $\phi_{x} \stackrel{\text { loc }}{\curvearrowright} \psi_{y}$ ) if there is a $g \in G_{x}$ such that $g\left[\left(\xi_{x}^{y} \circ \psi_{y}\right)(\gamma)\right] g^{-1}=\phi_{x}(\gamma)$. Then by [FS 2010, Lemma 2.7], there is a $\psi_{y^{\prime}} \in \mathscr{G}_{\psi_{y}}$ such that $\xi_{x}^{y} \circ \psi_{y}=\phi_{x}$. Extending this to an equivalence relation on $\mathscr{S}_{\mathscr{G}}^{\Gamma}$, we say that $\phi_{x} \approx \psi_{y}$ if there is a finite sequence of local coverings (in either direction) connecting an element of $\mathscr{G}_{\phi_{x}}$ to $\mathscr{G}_{\psi_{y}}$. We let $(\phi) \approx$ denote the $\approx$-class of $\phi$ and $T_{Q}^{\Gamma}$ denote the set of $\approx$-classes in $\mathscr{S}_{\mathscr{c}}^{\Gamma}$; when there is no risk of confusion, we denote the $\approx$-class of $\phi$ by $(\phi)$. The $\approx$-classes in $\mathscr{Y}_{\mathscr{G}}^{\Gamma}$ correspond exactly to the connected components of $\tilde{Q}_{\Gamma}$, so for each $(\phi) \in T_{Q}^{\Gamma}$, we let $\tilde{Q}_{(\phi)}$ denote the connected component consisting of

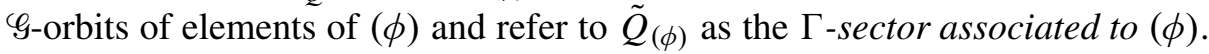

In [FS 2010, Lemma 2.5], we showed that a strong equivalence between orbifold groupoids induces a strong equivalence between their associated groupoids of $\Gamma$ sectors. Here, we are interested in foliation groupoids that are not necessarily étale.

Lemma 2.5. Suppose $\mathcal{G}$ and $\mathscr{G}^{\prime}$ are Morita equivalent orbifold groupoids. Then they are Morita equivalent via orbifold groupoids, that is, there is an orbifold groupoid $\mathscr{H}$ and strong equivalences

$$
\mathscr{G} \stackrel{e}{\longleftarrow} \mathscr{H} \stackrel{e^{\prime}}{\longrightarrow} \mathscr{G}^{\prime} .
$$

Of course, such an $\mathscr{H}$ always exists, and it is always a proper foliation groupoid. The point of this lemma is that $\mathscr{H}$ can taken to be étale. 
Proof. Choosing sufficiently small open covers of the spaces of objects consisting of linear orbifold charts, the groupoids $\mathscr{G}$ and $\mathscr{G}^{\prime}$ each give an orbifold atlas for the orbifold $Q$ presented by $G$ and $G^{\prime}$. These atlases need not be effective, but since they arose from orbifold groupoids, the kernels of the actions are appropriately restricted. Let $\mathscr{H}$ be the groupoid of the union of these two atlases; then there are clearly equivalences as required. These equivalences are strong, since the domains of charts from $\mathscr{G}$ and $\mathscr{G}^{\prime}$ are subsets of the space of objects of $\mathscr{H}$, so that the embeddings of these charts into the objects of $\mathscr{G}^{\prime}$ and $\mathscr{G}^{\prime}$ are surjective.

We fix some notation to distinguish between the structure maps and arrows of the groupoids we consider. We use $s, t, i, u$, and $m$ to denote the source, target, inverse, unit, and composition maps of a groupoid. Often, we suppress $m$ and express products multiplicatively by concatenation; that is, $m(a, b)=a b$. When it is helpful to distinguish between structure maps of groupoids, we will give them subscripts of the corresponding groupoid unless otherwise indicated. For a translation groupoid $\varphi_{\ltimes} \ltimes M$, we let $s_{\varphi_{\ltimes}}$ and $t_{\varphi_{\ltimes}}$ be the source and target maps, respectively, and let $(G \ltimes M)_{1}$ be the space of arrows. Note that $M$ is the space of objects. An arrow in $(\mathscr{G} \ltimes M)_{1}$ is given by a $g \in G_{1}$ and a $z \in M$ such that the anchor map sends $s(g)$ to $z$. We use $(g, z)$ to denote this arrow, so that $\operatorname{s\varphi }_{\ltimes} \times M(g, z)=z$ and $t \varphi_{\ltimes M}(g, z)=g z$. In particular, for the groupoid $\mathscr{G}^{\Gamma}=\mathscr{G}_{\ltimes} \mathscr{S}_{\mathscr{G}}^{\Gamma}$, an arrow is of the form $\left(g, \phi_{x}\right)$ with $s_{\varphi} \Gamma\left(g, \phi_{x}\right)=\phi_{x}$ and $t_{g} \Gamma\left(g, \phi_{x}\right)=g \phi_{x} g^{-1}$, so that $s(g)=x$ and $t(g)=g x$.

The following lemma will simplify many of our arguments; for the definitions, see [Adem et al. 2007, Definitions 2.14 and 2.15]. The proof is direct and omitted.

Lemma 2.6. Let $G$ be a groupoid, and let $M_{1}$ and $M_{2}$ be $G_{\text {-spaces }}$ with anchor maps $\alpha_{i}: M_{i} \rightarrow G_{0}$. Let $e_{0}: M_{1} \rightarrow M_{2}$ be a map that is $G$-equivariant, that is, $\alpha_{2} \circ e_{0}=\alpha_{1}$ and $e_{0}(h z)=h e_{0}(z)$ for each $z \in M_{1}$ and $h \in G_{1}$ with $s(h)=\alpha_{1}(z)$. Define $e_{1}:\left(\mathscr{G} \ltimes M_{1}\right)_{1} \rightarrow\left(G \ltimes M_{2}\right)_{1},(g, z) \mapsto\left(g, e_{0}(z)\right)$. Then $e_{0}$ is the map on objects and $e_{1}$ the map on arrows of a homomorphism $e: \mathscr{G} \ltimes M_{1} \rightarrow \mathscr{G} \ltimes M_{2}$ of groupoids. If $e_{0}$ is a bijection, then $e$ is an isomorphism.

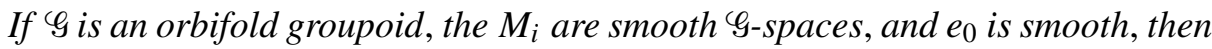
$e$ is a homomorphism of orbifold groupoids. If $e_{0}$ is a diffeomorphism, then $e$ is an isomorphism of orbifold groupoids.

\section{Connections between definitions of sectors}

Here, we compare the constructions of the $\Gamma$-sectors in Section 2 with one another, as well as with other constructions of sectors in the literature.

3A. Good orbifold. Let $Q$ be a good orbifold given by the quotient of a smooth manifold $M$ by a discrete group $G$ acting properly discontinuously. Then the translation groupoid $\mathscr{G}:=G \ltimes M$ is an orbifold groupoid presenting $Q$, and $Q$ admits two decompositions into $\Gamma$-sectors. 
As in Section 2A, we let $(M ; G)_{\Gamma}$ denote the space of $\Gamma$-sectors of $Q$ defined using the global $G$-action on $M$, that is,

$$
(M ; G)_{\Gamma}=\coprod_{(\phi) \in t_{M ; G}^{\Gamma}} C_{G}(\phi) \ltimes M^{\langle\phi\rangle} .
$$

As in Section 2B, we let $\tilde{Q}_{\Gamma}$ denote the space of $\Gamma$-sectors of $Q$ presented by $\varphi^{\Gamma}=\varphi_{\mathscr{C}} \ltimes \mathcal{S}_{\mathscr{G}}^{\Gamma}$. We claim the following.

Theorem 3.1. Let $Q$ be a good orbifold, so that $G=G \ltimes M$ is an orbifold groupoid presenting $Q$, and let $\Gamma$ be a finitely generated discrete group. Then $\varphi \varphi^{\Gamma}$ is isomorphic as an orbifold groupoid to $G \ltimes \coprod_{\phi \in \operatorname{Hom}(\Gamma, G)}\left(M^{\langle\phi\rangle}, \phi\right)$.

It follows that the spaces $(M ; G)_{\Gamma}$ and $\tilde{Q}_{\Gamma}$ are diffeomorphic as orbifolds. These spaces are not indexed in the same way; the set $t_{M ; G}^{\Gamma}$ is smaller than $T_{Q}^{\Gamma}$ whenever there is a homomorphism $\phi: \Gamma \rightarrow G$ such that $\sigma\left(M^{\langle\phi\rangle}\right)$ is not connected.

Example 3.2. Let $\mathbb{Z} / 3 \mathbb{Z}=\langle\alpha\rangle$ act on $S^{2}$ by rotations; the quotient orbifold $Q$ presented by $\mathbb{Z} / 3 \mathbb{Z} \ltimes S^{2}$ is a football with two singular points, $p_{s}$ and $p_{n}$, both of them with $\mathbb{Z} / 3 \mathbb{Z}$ isotropy. Let $\Gamma=\mathbb{Z}=\langle\gamma\rangle$, and define

$$
\phi_{0}, \phi_{1}, \phi_{2}: \mathbb{Z} \rightarrow \mathbb{Z} / 3 \mathbb{Z}, \quad \phi_{0}: \gamma \mapsto 1, \quad \phi_{1}: \gamma \mapsto \alpha, \quad \phi_{2}: \gamma \mapsto \alpha^{2} .
$$

Then the $\sim$-classes of the $\phi_{i}$ are the only elements of $t_{M ; G}^{\Gamma}$. Clearly, $(M ; G)_{\left(\phi_{0}\right)}$ is diffeomorphic to $Q$, and $(M ; G)_{\left(\phi_{1}\right)}$ and $(M ; G)_{\left(\phi_{2}\right)}$ are each diffeomorphic to $\left\{p_{s}, p_{n}\right\}$ with trivial $\mathbb{Z} / 3 \mathbb{Z}$-action.

Now, consider $\varphi_{\mathscr{C}} \ltimes \mathscr{S}_{\mathscr{G}}^{\Gamma}$. Let $\alpha_{s}$ generate $G_{p_{s}}$ and $\alpha_{n}$ generate $G_{p_{n}}$ for a choice of representatives of these isotropy groups. There are five $\approx$-classes of homomorphisms from $\Gamma$ into the local groups of $Q$ with the following representatives:

$$
\begin{aligned}
\psi_{0}: \mathbb{Z} \rightarrow G_{p}, & \gamma \mapsto 1 & \text { for all } p \in Q, & \\
\psi_{1, s}: \mathbb{Z} \rightarrow G_{p_{s}}, & \gamma \mapsto \alpha_{s}, & \psi_{2, s}: \mathbb{Z} \rightarrow G_{p_{s}}, & \gamma \mapsto \alpha_{s}^{2}, \\
\psi_{1, n}: \mathbb{Z} \rightarrow G_{p_{n}}, & \gamma \mapsto \alpha_{n}, & \psi_{2, n}: \mathbb{Z} \rightarrow G_{p_{n}}, & \gamma \mapsto \alpha_{n}^{2} .
\end{aligned}
$$

Then $\tilde{Q}_{\left(\psi_{0}\right)}$ is diffeomorphic to $Q$, while the sectors associated to each of the other four classes are given by a point with trivial $\mathbb{Z} / 3 \mathbb{Z}$-action.

Clearly, these two decompositions result in diffeomorphic orbifolds, although the individual sectors are indexed differently.

Proof of Theorem 3.1. Let $\mathscr{G}$ denote the translation groupoid $G \ltimes M$, so that $G_{0}=M$ and $G_{1}=G \times M$. Then $\mathscr{G}$ is an orbifold groupoid in the Morita equivalence class of orbifold structures for $Q$. Let $\zeta: G_{1}=G \times M \rightarrow G$ denote the projection onto the first factor. Then for each $\phi_{x} \in \mathscr{Y}_{\varphi}^{\Gamma}$, we have $\zeta \circ \phi_{x} \in \operatorname{Hom}(\Gamma, G)$, that is,

$$
\zeta \circ \phi_{x}: \Gamma \stackrel{\phi_{x}}{\longrightarrow}\left(G_{1}\right)_{x} \stackrel{\zeta}{\longrightarrow} G .
$$


We define the map

$$
\mathscr{L}: \mathscr{S}_{\mathscr{G}}^{\Gamma} \rightarrow \coprod_{\psi \in \operatorname{Hom}(\Gamma, G)}\left(M^{\langle\psi\rangle}, \psi\right), \quad \phi_{x} \mapsto\left(x, \zeta \circ \phi_{x}\right) \in\left(M^{\left\langle\zeta \circ \phi_{x}\right\rangle}, \zeta \circ \phi_{x}\right) .
$$

Then $\mathscr{L}$ is clearly injective; if $\mathscr{L}\left(\phi_{x}\right)=\mathscr{L}\left(\psi_{y}\right)$, then $\left(x, \zeta \circ \phi_{x}\right)=\left(y, \zeta \circ \psi_{y}\right)$, so that $x=y$ and $\phi_{x}=\psi_{y}$. To show that $\mathscr{L}$ is surjective, let $(x, \psi) \in\left(M^{\langle\psi\rangle}, \psi\right)$ for some $\psi \in \operatorname{Hom}(\Gamma, G)$ and define $\psi_{x}: \Gamma \rightarrow \mathscr{G}_{\text {by }} \psi_{x}(\gamma)=(\psi(\gamma), x)$. Then clearly $\mathscr{L}\left(\psi_{x}\right)=(x, \psi)$, and $\mathscr{E}$ is a bijection. Moreover, given a chart $\kappa_{\phi_{x}}: V_{x}^{\left\langle\phi_{x}\right\rangle} \rightarrow \mathscr{S}_{\mathscr{G}}^{\Gamma}$ for $\mathscr{Y}_{\mathscr{G}}^{\Gamma}$ near $\phi_{x}$, we have that

$$
M^{\left\langle\phi_{x}\right\rangle} \supseteq V_{x}^{\left\langle\phi_{x}\right\rangle} \stackrel{\kappa_{\phi_{x}}}{\longrightarrow} \mathscr{S}_{\varphi}^{\Gamma} \stackrel{\mathscr{L}}{\longrightarrow}\left(M^{\left\langle\zeta \circ \phi_{x}\right\rangle}, \zeta \circ \phi_{x}\right)
$$

is simply the identity on $V_{x}^{\left\langle\phi_{x}\right\rangle}$. It follows that $\mathscr{E}$ is smooth with smooth inverse, and hence a diffeomorphism.

The anchor map of the $\mathscr{G}_{\text {-action on }} \mathscr{Y}_{\mathscr{G}}^{\Gamma}$ is $\beta_{\Gamma}: \mathscr{Y}_{\mathscr{G}}^{\Gamma} \rightarrow M$, with $\beta_{\Gamma}: \phi_{x} \mapsto x$. Let $\alpha: \coprod_{\psi \in \operatorname{Hom}(\Gamma, G)}\left(M^{\langle\psi\rangle}, \psi\right) \rightarrow M$ be defined by $\alpha:(x, \phi) \mapsto x$; then $\alpha$ is the anchor map of a $\mathscr{G}_{\text {-action on }} \coprod_{\psi \in \operatorname{Hom}(\Gamma, G)}\left(M^{\langle\psi\rangle}, \psi\right) \rightarrow M$ defined by

$$
(g, x)(x, \phi)=\left(g x, g \phi g^{-1}\right)
$$

that clearly coincides with the $G$-action. Hence, we need only note that for each $(g,(x, \phi)) \in G \times \coprod_{\psi \in \operatorname{Hom}(\Gamma, G)}\left(M^{\langle\psi\rangle}, \psi\right)$ and $\phi_{x} \in \mathscr{Y}_{\varphi}^{\Gamma}$ given by $\gamma \mapsto(\phi(\gamma), x)$ (so that $\left.\mathscr{L}\left(\phi_{x}\right)=(x, \phi)\right)$,

$$
\begin{aligned}
(g,(x, \phi)) \mathscr{L}\left(\phi_{x}\right) & =(g,(x, \phi))\left(x, \zeta \circ \phi_{x}\right)=(g,(x, \phi))(x, \phi) \\
& =\left(g x, g \phi g^{-1}\right)=\mathscr{E}\left[\left(g, \phi_{x}\right) \phi_{x}\right],
\end{aligned}
$$

and then $\mathscr{L}$ is $\mathscr{G}$-equivariant. It follows by Lemma 2.6 that $\mathscr{L}$ is the map on objects of an isomorphism of Lie groupoids.

By Lemma 2.3, $G \ltimes \coprod_{\phi \in \operatorname{Hom}(\Gamma, G)}\left(M^{\langle\phi\rangle}, \phi\right)$ and $(M ; G)_{\Gamma}$ are Morita equivalent. Hence, by virtue of [FS 2010, Lemma 2.5] and Lemma 2.5 above, we have the following.

Corollary 3.3. Let $Q$ be a good orbifold presented by $G \ltimes M$, with $G$ discrete, and let $\Gamma$ be a finitely generated discrete group. If $G$ is any orbifold groupoid that presents $Q$, then $(M ; G)_{\Gamma}$ and $\varphi \varphi^{\Gamma}$ are Morita equivalent. Hence, the two definitions of $\Gamma$-sectors coincide. In particular, $(M ; G)_{\Gamma}$ is Morita equivalent to an orbifold groupoid.

Finally, the proof of Theorem 3.1 generalizes readily to proper étale orbispaces.

Theorem 3.4. Let $Y$ be a $T_{1} G$-space with $G$ discrete such that the isotropy group of each point is finite, let $\Gamma$ be a finitely generated discrete group, and let $\mathscr{G}=G \ltimes Y$. Then $G^{\Gamma}$ is isomorphic as a topological groupoid to $G \ltimes \bigsqcup_{\phi \in \operatorname{Hom}(\Gamma, G)}\left(M^{\langle\phi\rangle}, \phi\right)$. 
Proof. Algebraically, the proof is identical to that of Theorem 3.1. Based on the note after Definition 2.4, the map $\mathscr{L}$ is clearly a homeomorphism. The induced map on arrows given by Lemma 2.6 is clearly a homeomorphism as well.

3B. Quotient orbifolds. In the case where $G$ is not discrete, we have:

Theorem 3.5. Let $G$ be a Lie group that acts smoothly on the smooth manifold $M$ satisfying conditions (i), (ii), and (iii), so that $G \ltimes M$ presents an orbifold $Q$. Let $G$ be an orbifold groupoid representing $Q$, so that $G \ltimes M$ and $\varphi$ are Morita equivalent. Then $\varphi^{\Gamma}$ and $(M ; G)_{\Gamma}$ are Morita equivalent.

Proof. First, we construct a specific orbifold groupoid Morita equivalent to $G \ltimes M$.

If $G$ acts properly on $M$ with discrete isotropy groups, then $M$ is foliated by (connected components of) $G$-orbits [Moerdijk and Mrčun 2003, page 16]. Pick $x \in M$; then there is a unique $G_{x}$-space $S_{x}$ and a $G$-diffeomorphism of $G \times_{G_{x}} S_{x}$ onto an open subset of $M$ containing $x$. We recall the construction of $G \times{ }_{G_{x}} S_{x}$. If $(u, y) \in G \times S_{x}$ and $k \in G_{x}$, then $k(u, y)=\left(u k^{-1}, k y\right)$ defines a $G_{x}$-action on $G \times S_{x}$, and $G \times{ }_{G_{x}} S_{x}$ is the orbit space of this action. Then the $G$-action on $G \times S_{x}$ given by $g^{\prime}(g, y)=\left(g^{\prime} g, y\right)$ induces a $G$-action on $G \times{ }_{G_{x}} S_{x}$ [tom Dieck 1987, page 32]. In particular, the slice $S_{x}$ is a transversal for the foliation of $\left(G \times_{G_{x}} S_{x}\right)$ by $G$-orbits. We note that $S_{x}$ is not a complete transversal unless $G / G_{x}$ is connected; in general, a complete transversal to the foliation of $\left(G \times{ }_{G_{x}} S_{x}\right)$ can be formed by picking one translate $g S_{x}$ of the slice of $S_{x}$ in each connected component of $\left(G \times{ }_{G_{x}} S_{x}\right)$.

Since $M / G$ is paracompact, an open cover of $M / G$ formed by picking a chart of the form $G \times_{G_{x}} S_{x}$ for a choice of one point $x$ in each $G$-orbit of $M$ can be refined to a locally finite cover by shrinking the $S_{x}$; hence, we can form a complete transversal $S$ to the foliation of $M$ by $G$-orbits by taking the (possibly disconnected) union of slices $S_{x}$.

By [Crainic and Moerdijk 2001, Theorem 1 and Lemma 2], $G \ltimes M$ is equivalent to the groupoid given by the restriction $\left.(G \ltimes M)\right|_{S}$ of $G \ltimes M$ to a complete transversal $S$ (note that the essential equivalence of [Crainic and Moerdijk 2001] corresponds to an equivalence in [Adem et al. 2007, Definition 1.42]; we use the language of the latter for consistency). Moreover, $\left.(G \ltimes M)\right|_{S}$ is étale. Since $G \ltimes M$ is proper and properness is preserved under equivalence, $\left.(G \ltimes M)\right|_{S}$ is an orbifold groupoid.

The next argument follows [Adem and Ruan 2003, Theorem 5.3], which treats the case of $\Gamma=\mathbb{Z}$.

Pick a homomorphism $\phi: \Gamma \rightarrow G$ with nonempty fixed-point set in $M$. Since $G$ acts on $M$ with discrete isotropy, $C_{G}(\phi)$ clearly acts on $M^{\langle\phi\rangle}$ with discrete isotropy and hence foliates $M^{\langle\phi\rangle}$ by (connected components of) $C_{G}(\phi)$-orbits. We construct a complete transversal to this foliation from the complete transversal $S$. 
Pick a chart of the form $\left(G \times_{G_{x}} S_{x}\right)$ where the slice $S_{x}$ is contained in $S$. Then $\left(G \times{ }_{G_{x}} S_{x}\right)^{\langle\phi\rangle}$ is by definition the set of $G_{x}(u, y) \in\left(G \times{ }_{G_{x}} S_{x}\right)$ such that for all $\gamma \in \Gamma$, there exists $h \in G_{x}$ such that $(\phi(\gamma) u, y)=h(u, y)$, where again $h(u, y)=\left(u h^{-1}, h y\right)$. We claim that $\left(G \times_{G_{x}} S_{x}\right)^{\langle\phi\rangle}$ is given by

$$
\left\{G_{x}(u, y) \in\left(G \times_{G_{x}} S_{x}\right): u^{-1}(\operatorname{Im} \phi) u \leq G_{x}, y \in S_{x}^{\left\langle u^{-1} \phi u\right\rangle}\right\} .
$$

Suppose $u^{-1}(\operatorname{Im} \phi) u \leq G_{x}$ and $y \in S_{x}^{\left\langle u^{-1} \phi u\right\rangle}$. For each $\gamma \in \Gamma$,

$$
\begin{aligned}
u^{-1} \phi(\gamma)^{-1} u(u, y) & =\left(u\left(u^{-1} \phi(\gamma)^{-1} u\right)^{-1}, u^{-1} \phi(\gamma)^{-1} u y\right) \\
& =\left(u u^{-1} \phi(\gamma) u, y\right)=(\phi(\gamma) u, y) .
\end{aligned}
$$

Since $u^{-1} \phi(\gamma)^{-1} u \in G_{x}$, it follows that the $G_{x}$-orbits $G_{x}(\phi(\gamma) u, y)=G_{x}(u, y)$. This is true for each $\gamma \in \Gamma$, so $G_{x}(u, y) \in\left(S_{x} \times_{G_{x}} G\right)^{\langle\phi\rangle}$.

Conversely, suppose the orbit $G_{x}(u, y)$ is fixed by $\phi(\gamma)$ for each $\gamma \in \Gamma$. Then for each $\gamma \in \Gamma$, there is an $h \in G_{x}$ such that $(\phi(\gamma) u, y)=h(u, y)=\left(u h^{-1}, h y\right)$. It follows that $\phi(\gamma) u=u h^{-1}$, so that $u^{-1} \phi(\gamma) u=h^{-1} \in G_{x}$. Also, $y=h y$ so that

$$
y \in S_{x}^{\langle h\rangle}=S_{x}^{\left\langle u^{-1} \phi(\gamma)^{-1} u\right\rangle} .
$$

This is true for each $\gamma \in \Gamma$, so $u^{-1}(\operatorname{Im} \phi) u \leq G_{x}$ and $y \in S_{x}^{\left\langle u^{-1} \phi u\right\rangle}$, proving the expression in (3-1) of $\left(G \times{ }_{G_{x}} S_{x}\right)^{\langle\phi\rangle}$.

Now, let $\mathrm{O}_{\phi}$ be the collection of $\psi: \Gamma \rightarrow G_{x} \leq G$ that are conjugate to $\phi$ in $G$. Then $G_{x}$ acts on $\bigsqcup_{\psi \in \mathcal{O}_{\phi}}\left(S_{x}^{\langle\psi\rangle}, \psi\right)$ via $h(y, \psi)=\left(h y, h \psi h^{-1}\right)$. We let $[y, \psi]$ denote the $G_{x}$-orbit of $(y, \psi)$. Define the map

$$
\mathscr{E}:\left(G \times_{G_{x}} S_{x}\right)^{\langle\phi\rangle} \rightarrow\left(\coprod_{\psi \in \mathbb{O}_{\phi}}\left(S_{x}^{\langle\psi\rangle}, \psi\right)\right) / G_{x}, \quad G_{x}(u, y) \mapsto\left[y, u^{-1} \phi u\right] .
$$

This map is well defined, since for $h \in G_{x}$,

$$
\begin{aligned}
\mathscr{E}\left(G_{x} h(u, y)\right) & =\mathscr{E}\left(G_{x}\left(u h^{-1}, h y\right)\right)=\left[h y, h u^{-1} \phi u h^{-1}\right] \\
& =h\left[y, u^{-1} \phi u\right]=\left[y, u^{-1} \phi u\right]=\mathscr{E}(u, y) .
\end{aligned}
$$

Note that $y \in S_{x}^{\left\langle u^{-1} \phi u\right\rangle}$ whenever $G_{x}(u, y) \in\left(G \times_{G_{x}} S_{x}\right)^{\langle\phi\rangle}$, and note further that the map $\mathscr{E}$ is clearly smooth, both observations by virtue of (3-1).

The map $\mathscr{E}$ is not injective. However, we claim that $\mathscr{E}\left(G_{x}(u, y)\right)=\mathscr{E}\left(G_{x}\left(v, y^{\prime}\right)\right)$ if and only if there is a $z \in C_{G}(\phi)$ such that $z\left(G_{x}(u, y)\right)=G_{x}\left(v, y^{\prime}\right)$, that is, $(z u, y)=h\left(v, y^{\prime}\right)=\left(v h^{-1}, h y^{\prime}\right)$ for some $h \in G_{x}$. If this is the case, then

$$
(u, y)=\left(z^{-1} v h^{-1}, h y^{\prime}\right),
$$


so that

$$
\begin{aligned}
\mathscr{E}\left(G_{x}(u, y)\right) & =\left[y, u^{-1} \phi u\right]=\left[h y^{\prime},\left(z^{-1} v h^{-1}\right)^{-1} \phi z^{-1} v h^{-1}\right] \\
& =\left[h y^{\prime}, h v^{-1} z \phi z^{-1} v h^{-1}\right]=h\left[y^{\prime}, v^{-1} \phi v\right] \\
& =\left[y^{\prime}, v^{-1} \phi v\right]=\mathscr{E}\left(G_{x}\left(v, y^{\prime}\right)\right) .
\end{aligned}
$$

Conversely, if $\mathscr{E}\left(G_{x}(u, y)\right)=\mathscr{E}\left(G_{x}\left(v, y^{\prime}\right)\right)$, then $\left[y, u^{-1} \phi u\right]=\left[y^{\prime}, v^{-1} \phi v\right]$, so that there is an $h \in G_{x}$ such that $\left(y, u^{-1} \phi u\right)=h\left(y^{\prime}, v^{-1} \phi v\right)=\left(h y^{\prime}, h v^{-1} \phi v h^{-1}\right)$. It follows that $y=h y^{\prime}$ and $u^{-1} \phi u=h v^{-1} \phi v h^{-1}$, that is, that $\phi=v h^{-1} u^{-1} \phi u h v^{-1}$. Hence, letting $z=v h^{-1} u^{-1}$, we have that $z \in C_{G}(\phi)$ and $z u=v h^{-1}$, so that $(z u, y)=\left(v h^{-1}, h y^{\prime}\right)$.

To see that this map is surjective, let

$$
[y, \psi] \in \coprod_{\psi \in \mathbb{O}_{\phi}}\left(S_{x}^{\langle\psi\rangle}, \psi\right)
$$

then there is a $u \in G$ such that $u \psi u^{-1}=\phi$. Then $(u, y) \in\left(G \times{ }_{G_{x}} S_{x}\right)^{\langle\phi\rangle}$ and $\mathscr{E}\left(G_{x}(u, y)\right)=[y, \psi]$.

With this, we see that $\mathscr{E}$ induces a diffeomorphism from $\left(G \times{ }_{G_{x}} S_{x}\right)^{\langle\phi\rangle} / C_{G}(\phi)$ onto $\left(\coprod_{\psi \in \mathcal{O}_{\phi}}\left(S_{x}^{\langle\psi\rangle}, \psi\right)\right) / G_{x}$. Let $\left(\psi{\underset{G}{G_{x}}}\right.$ denote the $G_{x}$-conjugacy class of $\psi$, to distinguish it from the $G$-conjugacy class. Recall from the proof of Lemma 2.3 that the strong equivalence

$$
G_{x} \ltimes \coprod_{\psi \in \operatorname{Hom}\left(\Gamma, G_{x}\right)}\left(S_{x}^{\langle\psi\rangle}, \psi\right) \rightarrow \coprod_{(\psi)_{G_{x} \in \mathbb{O}_{\phi} / G_{x}}} C_{G_{x}}(\psi) \ltimes S_{x}^{\langle\psi\rangle}
$$

restricts to an equivalence

$$
G_{x} \ltimes \coprod_{\psi_{0} \in(\psi)_{G_{x}}}\left(S_{x}^{\langle\psi\rangle}, \psi\right) \rightarrow C_{G_{x}}(\psi) \ltimes S_{x}^{\langle\psi\rangle}
$$

for each $\stackrel{G_{x}}{\sim}$-class $(\psi)_{G_{x}}$. Noting that $O_{\phi}$ clearly consists of entire $\stackrel{G_{x}}{\sim}$-classes, we have that there is an equivalence

$$
G_{x} \ltimes \coprod_{\psi \in \mathscr{O}_{\phi}}\left(S_{x}^{\langle\psi\rangle}, \psi\right) \rightarrow \coprod_{(\psi)_{G_{x} \in \mathbb{O}_{\phi} / G_{x}}} C_{G_{x}}(\psi) \ltimes S_{x}^{\langle\psi\rangle},
$$

where the $G_{x}$-action on $\sigma_{\phi}$ is by conjugation. This implies that there is a diffeomorphism

$$
\left(\coprod_{\psi \in \mathbb{O}_{\phi}}\left(S_{x}^{\langle\psi\rangle}, \psi\right)\right) / G_{x} \rightarrow \coprod_{(\psi)_{G_{x} \in \mathscr{O}_{\phi} / G_{x}}} S_{x}^{\langle\psi\rangle} / C_{G_{x}}(\psi) .
$$


Note that $\left(G \times_{G_{x}} S_{x}\right)^{\langle\phi\rangle}$ is empty unless $\phi$ is conjugate in $G$ to a homomorphism with image in $G_{x}$. Choose one representative $\psi$ from each $G_{x}$-conjugacy class $(\psi)_{G_{x}}$. Recall that the map $\mathscr{E}$ is constant on $C_{G}(\phi)$-orbits. From its definition, $\mathscr{E}$ maps the submanifold $S_{x}^{\langle\psi\rangle}$ of the slice $S_{x}$ to the $G_{x}$-orbit of $\left(S_{x}^{\langle\psi\rangle}, \psi\right)$. Moreover, if $\psi$ is the chosen representative of the conjugacy class $(\psi)_{G_{x}}$, the equivalence in Lemma 2.3 maps $\left(S_{x}^{\langle\psi\rangle}, \psi\right)$ onto $S_{x}^{\langle\psi\rangle}$. It follows from this diffeomorphism and these observations that the disjoint union

$$
\mathscr{S}_{x}=\coprod_{(\psi)_{G_{x} \in \mathbb{O}_{\phi} / G_{x}}} S_{x}^{\langle\psi\rangle}
$$

is a complete transversal to the foliation of $\left(G \times{ }_{G_{x}} S_{x}\right)^{\langle\phi\rangle}$ by connected components of $C_{G}(\phi)$-orbits. Forming $\mathscr{Y}_{x}$ for each chart for $S$ as above, the (possibly disconnected) union $\tilde{S}$ of the $\mathscr{S}_{x}$ forms a complete transversal to the foliation of $M^{\langle\phi\rangle}$ by the $C_{G}(\phi)$-action.

As usual, let $\left.(G \ltimes M)\right|_{S} ^{\Gamma}$ denote the groupoid of $\Gamma$-sectors for the orbifold groupoid $\left.(G \ltimes M)\right|_{S}$, constructed as in Section 2B. Note that the space of objects of $\left.(G \ltimes M)\right|_{S}$ is simply $S$, while the arrows of $\left.(G \ltimes M)\right|_{S}$ are given by $(g, x) \in G \times S$ such that $g x \in S$. Clearly, then, the isotropy group of a point $x \in S$ is simply $G_{x}$, the isotropy group of $x$ as a point in $M$. It follows that the space of objects of $\left.(G \ltimes M)\right|_{S} ^{\Gamma}$ is the set of homomorphisms $\psi_{x}: \Gamma \rightarrow G_{x}$ for $x \in S$ with local charts given by $V_{x}^{\left\langle\psi_{x}\right\rangle}$. Since the action of an arrow $(g, x)$ in $\left.(G \ltimes M)\right|_{S}$ is given by $g \phi_{x} g^{-1}$, yielding a homomorphism from $\Gamma$ into $G_{g x}$, the groupoid $\left.(G \ltimes M)\right|_{S} ^{\Gamma}$ is isomorphic to the restriction of the groupoid $C_{G}(\phi) \ltimes M^{\langle\phi\rangle}$ to the complete transversal $\tilde{S}$ given above. This is true for each $(\phi) \in t_{M ; G}^{\Gamma}$, so there is an equivalence from $\left.(G \ltimes M)\right|_{S} ^{\Gamma}$ to $G \ltimes \bigsqcup_{\phi \in \operatorname{Hom}(\Gamma, G)} M^{\langle\phi\rangle}$. Hence $\left.(G \ltimes M)\right|_{S} ^{\Gamma}$ and $(M ; G)_{\Gamma}$ are Morita equivalent by Lemma 2.3.

To complete the proof, suppose $\mathscr{G}$ is any orbifold groupoid Morita equivalent to $G \ltimes M$. Then $G$ is Morita equivalent to $\left.(G \ltimes M)\right|_{S}$ via étale groupoids by Lemma 2.5, implying by [FS 2010, Lemma 2.5] that the $\Gamma$-sectors of the two groupoids are Morita equivalent.

Corollary 3.6. Let $G$ be a Lie group that acts smoothly on the smooth manifold $M$ satisfying conditions (i), (ii), and (iii), so that $G \ltimes M$ presents an orbifold $Q$. Let $\Gamma$ be a finitely generated discrete group. Then $(M ; G)_{\Gamma}$ is Morita equivalent to an orbifold groupoid and hence presents an orbifold.

Though Example 3.2 shows the correspondence $T_{Q}^{\Gamma} \ni\left(\phi_{x}\right) \approx \mapsto\left(\zeta \circ \phi_{x}\right) \sim \in t_{M ; G}^{\Gamma}$ is not injective, it is clearly surjective. By Theorems 3.1 and 3.5 and the fact that $\approx$-classes are precisely connected components of $\tilde{Q}_{\Gamma}=\left|\varphi^{\Gamma}\right|$, it is clear that each $\approx$-class corresponds to a connected component of a $\sim$-class of $(M ; G)_{\Gamma}$. 
With this, we note that the equivalence $\approx$ defined in Section $2 \mathrm{~B}$ on objects of $\varphi^{\Gamma}$ can be expressed naturally on either model of $(M ; G)_{\Gamma}$. Using the groupoid $(M ; G)_{\Gamma}$ of Definition 2.1, we say $(x, \phi) \approx(y, \phi)$ for $(x, \phi),(y, \phi) \in M^{\langle\phi\rangle}$ if the orbits $C_{G}(\phi) x$ and $C_{G}(\phi) y$ are on the same connected component of $M^{\langle\phi\rangle} / C_{G}(\phi)$. Similarly, using the Morita equivalent groupoid representing $(M ; G)_{\Gamma}$ given by Lemma 2.3 , we say that $(x, \phi) \approx\left(y, \phi^{\prime}\right)$ for two points

$$
(x, \phi),\left(y, \phi^{\prime}\right) \in \coprod_{\psi \in \operatorname{Hom}(\Gamma, G)}\left(M^{\langle\psi\rangle}, \psi\right)
$$

whenever there is a $g \in G$ such that $g \phi^{\prime} g^{-1}=\phi$ and such that the orbits $G(x, \phi)$ and $G\left(y, \phi^{\prime}\right)=G(g y, \phi)$ are on the same connected component of

$$
\left(\coprod_{\psi \in \operatorname{Hom}(\Gamma, G)}\left(M^{\langle\psi\rangle}, \psi\right)\right) / G
$$

Clearly, the three definitions of $\approx$ coincide in that they define the same equivalence classes on the quotient space, and the $\approx$-classes correspond exactly to connected components. We let $(x, \phi) \approx$ denote the $\approx$-class of the point $(x, \phi)$ in either case and $T_{M ; G}^{\Gamma}$ the set of $\approx$-classes. Then $T_{M ; G}^{\Gamma}$ and $T_{Q}^{\Gamma}$ obviously coincide.

In the same way, the definitions in [FS 2010, Section 3] can be reformulated from the perspective of a presentation as a quotient. Let $(x, \phi) \approx,(y, \psi) \approx \in T_{M ; G}^{\Gamma}$ and let $\left(M^{\langle\phi\rangle} / C_{G}(\phi)\right)_{x}$ and $\left(M^{\langle\psi\rangle} / C_{G}(\psi)\right)_{y}$ denote the connected components of $M^{\langle\phi\rangle} / C_{G}(\phi)$ and $M^{\langle\psi\rangle} / C_{G}(\psi)$ containing the orbits of $x$ and $y$, respectively. We say that $(x, \phi) \approx \leq(y, \psi) \approx$ if

$$
\pi\left(\left(M^{\langle\phi\rangle} / C_{G}(\phi)\right)_{x}\right) \subseteq \pi\left(\left(M^{\langle\psi\rangle} / C_{G}(\phi)\right)_{y}\right),
$$

where $\pi:(M ; G)_{\Gamma} \rightarrow M / G$ denotes the map $C_{G}(\phi)(x, \phi) \mapsto G x$. Similarly, $\Gamma$ covers the local groups of $Q$ if, for every $H \leq G$ such that $M^{H} \neq \varnothing$, there is a surjective homomorphism $\phi: \Gamma \rightarrow H$.

3C. Connections between $\Gamma$-sectors and other sectors. The definition of the $\Gamma$ sectors was motivated by that of the inertia orbifold and the $k$-multisectors given in [Adem et al. 2007, pages 52-53]; see also [Chen and Ruan 2004]. Hence, the $\Gamma$-sectors generalize the definition of the multisectors, as follows.

Proposition 3.7. Let $Q$ be an orbifold presented by the orbifold groupoid $G$ and let $\mathbb{F}_{k}$ denote the free group on l-generators. The groupoids $\varphi_{\mathscr{G}} \ltimes \mathscr{Y}_{\mathscr{G}}^{k}$ and $\varphi_{\ltimes} \ltimes \mathscr{Y}_{\mathscr{G}}^{\mathbb{F}_{k}}$ are isomorphic, and $\tilde{Q}_{\mathbb{F}_{k}}$ is diffeomorphic to the space of $k$-multisectors $\tilde{Q}_{k}$.

Proof. This follows almost immediately from the definition. Let $\mathbb{F}_{k}$ be generated by $\gamma_{1}, \ldots, \gamma_{k}$, and recall from [Adem et al. 2007] that $\mathscr{S}_{\mathscr{g}}^{k}$ is defined to be the set

$$
\left\{\left(g_{1}, \ldots, g_{k}\right): g_{i} \in G_{1}, s\left(g_{i}\right)=t\left(g_{j}\right) \text { for all } i, j \leq k\right\} .
$$


To each $\left(g_{1}, \ldots, g_{k}\right) \in \mathscr{S}_{\mathscr{G}}^{k}$ with $s\left(g_{i}\right)=t\left(g_{j}\right)=x$, there is a unique homomorphism $\phi_{x}: \mathbb{F}_{k} \rightarrow G_{x}$ such that $\phi_{x}\left(\gamma_{i}\right)=g_{i}$. It is obvious that the identification $\left(g_{1}, \ldots, g_{k}\right) \mapsto \phi_{x}$ is a homeomorphism $\mathscr{Y}_{\mathscr{G}}^{k} \rightarrow \mathscr{Y}_{\mathscr{G}}^{\mathbb{F}_{k}}$. With this, we need only note that the action of $\mathscr{G}_{\text {on }} \mathscr{S}_{\mathscr{G}}^{k}$ and $\mathscr{S}_{\mathscr{G}}^{\mathbb{F}_{k}}$ are defined identically, and hence the result follows by an application of Lemma 2.6.

Corollary 3.8. Let 9 be an orbifold groupoid. Then $9 \mathbb{Z}$ is isomorphic as a groupoid to the inertia groupoid $\Lambda \mathscr{G}$. In particular, the space of $\mathbb{Z}$-sectors $\tilde{Q}_{\mathbb{Z}}$ is diffeomorphic to the inertia orbifold $\tilde{Q}$.

Leida [2005] defines the fixed-point sectors of an orbifold groupoid $\mathscr{G}$. Recall that Leida defines $\tilde{\mathscr{S}}(\mathscr{G})=\left\{(x, H) \mid x \in G_{0}, H \leq G_{x}\right\}$, and $\tilde{\mathscr{G}}=\mathscr{G} \ltimes \tilde{\mathscr{S}}(\mathscr{G})$. Similarly, for each subgroup $H$ of $G_{1}, \tilde{\mathscr{Y}}^{H}(\mathscr{\varphi})$ is the subset $\{(x, K) \mid K \cong H\}$. Define the map $\varrho: \mathscr{S}_{\varphi}^{\Gamma} \rightarrow \tilde{\mathscr{S}}(\varphi), \phi_{x} \mapsto\left(x, \operatorname{Im} \phi_{x}\right)$. For each point $\left(x, \operatorname{Im} \phi_{x}\right)=\varrho\left(\phi_{x}\right)$ in the image of $\varrho$, there is a neighborhood $V_{x}$ of $x$ in $G_{0}$ such that the restriction $\left.\mathscr{G}\right|_{V_{x}}$ is isomorphic to $G_{x} \ltimes V_{x}$. This corresponds to a neighborhood of $\left(x, \operatorname{Im} \phi_{x}\right)$ in $\tilde{\mathscr{S}} \operatorname{Im} \phi_{x}(\mathscr{G})$ diffeomorphic to $V_{x}^{\left\langle\phi_{x}\right\rangle}$ such that the restriction of $\tilde{\mathscr{G}}^{\operatorname{Im} \phi_{x}}$ is isomorphic to $N_{G_{x}}\left(\operatorname{Im} \phi_{x}\right) \ltimes V_{x}^{\left\langle\phi_{x}\right\rangle}$ [Leida 2005, Section 2.2]; $N_{G_{x}}\left(\operatorname{Im} \phi_{x}\right)$ denotes the normalizer of $\operatorname{Im} \phi_{x}$ in $G_{x}$. Similarly, there is a neighborhood of $\phi_{x}$ in $\mathcal{G}_{\mathscr{G}}^{\Gamma}$ such that the restriction of $\varphi^{\Gamma}$ is isomorphic to $C_{G_{x}}\left(\phi_{x}\right) \ltimes V_{x}^{\left\langle\phi_{x}\right\rangle}$. When restricted to these neighborhoods, the map $\varrho$ is simply the embedding of $C_{G_{x}}\left(\phi_{x}\right) \ltimes V_{x}^{\left\langle\phi_{x}\right\rangle}$ into $N_{G_{x}}\left(\phi_{x}\right) \ltimes V_{x}^{\left\langle\phi_{x}\right\rangle}$. If $\psi_{x}$ is another point with $\varrho\left(\psi_{x}\right)=\left(x, \operatorname{Im} \phi_{x}\right)$, then $\operatorname{Im} \psi_{x}=\operatorname{Im} \phi_{x}$, so that

$$
C_{G_{x}}\left(\psi_{x}\right) \ltimes V_{x}^{\left\langle\psi_{x}\right\rangle}=C_{G_{x}}\left(\phi_{x}\right) \ltimes V_{x}^{\left\langle\phi_{x}\right\rangle} .
$$

If a point $\left(x, \operatorname{Im} \phi_{x}\right)$ is in the image of $\varrho$, then every point in $\tilde{\mathscr{G}} \operatorname{Im} \phi_{x}$ is in the

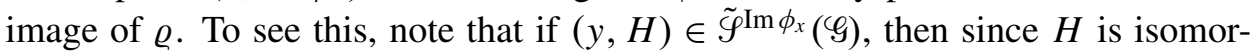
phic to $\operatorname{Im} \phi_{x}$, there is a homomorphism $\psi_{y}: \Gamma \rightarrow G_{y}$ with image $H$. It follows that $\varrho\left(\psi_{y}\right)=(y, H)$. It need not be the case that $\psi_{y} \approx \phi_{x}$. However, using the techniques of [FS 2010, Lemma 3.2], it is easy to see that the images of $\Gamma$-sectors $(\phi)$ via $\varrho$ are entire connected components of $\tilde{\mathscr{G}}$. In particular, if $\left(x, \operatorname{Im} \phi_{x}\right)$ and $(y, H)$ are in the same connected component of a fixed-point sector $\tilde{g}^{\operatorname{Im} \phi_{x}}=\tilde{\varphi}^{H}$, then they are connected by a path in $\tilde{g}^{H}$ and hence a finite number of charts of the form $N_{G_{x_{i}}}\left(H_{i}\right) \ltimes V_{x_{i}}^{H_{i}}$ with each $H_{i}$ isomorphic. Arrows $g \in G_{1}$ connecting orbits of points in $V_{x_{i}}^{H_{i}}$ to those in $V_{x_{i+1}}^{H_{i+1}}$ act on homomorphisms with images in $H_{i}$, resulting in homomorphisms with images in $H_{i+1}$. Therefore, a sequence of homomorphisms $\phi_{x_{i}}$ can be defined in each chart, showing that there is a $\phi_{y} \approx \phi_{x}$ with $\varrho\left(\phi_{y}\right)=(y, H)$.

If $\Gamma$ covers the local groups of $Q$ - that is, if for each subgroup $H \leq G_{x}$ of an isotropy group of $Q$, there is a surjective homomorphism $\Gamma \rightarrow H$ - then it is clear that each $(x, H)$ is the image via $\varrho$ of a $\phi_{x}$ with $\operatorname{Im} \phi_{x}=H$. Hence, $\varrho$ is surjective, and we have the following. 
Proposition 3.9. Let $\Gamma$ be a finitely generated discrete group and $\mathscr{G}$ an orbifold groupoid presenting the orbifold $Q$. Each $\Gamma$-sector of $Q$ is an orbifold cover of a connected component of each $\tilde{\varphi} \operatorname{Im} \phi_{x}(G)$. If $\phi_{x}$ is chosen to have minimal isotropy in $(\phi)$, then the $\Gamma$-sector $(\phi)$ is a $\left[N_{G_{x}}\left(\phi_{x}\right): C_{G_{x}}\left(\phi_{x}\right)\right]$-cover of the corresponding fixed-point sector. If $\Gamma$ covers the local groups of $Q$, then each connected component of each $\tilde{G}^{H}$ is orbifold-covered by a $\Gamma$-sector.

Since the homotopy groups of an orbifold groupoid $\mathscr{G}$ are Morita invariant, the homotopy groups of the $\Gamma$-sectors are Morita invariants for each finitely generated discrete group $\Gamma$. See [Leida 2005; Chen 2006; Henriques and Gepner 2007] for more on homotopy theory and homotopy groups of orbifolds and orbispaces.

\section{A model of the $\Gamma$-sectors using generalized loop spaces}

In [LU 2002], it is shown that the inertia orbifold of an orbifold $Q$ occurs in the context of the loop space of $Q$. It appears as the subset of constant loops or equivalently the set of loops fixed by the natural $S^{1}$-action on the loop space. In this section, we show how this construction can be generalized to demonstrate that the $\Gamma$-sectors of an orbifold arise in the same way when considering maps from a closed manifold $M_{\Gamma}$ with fundamental group $\Gamma$. See [Tamanoi 2003] for similar results for global quotients from a different perspective.

Many of the results in this section can be proved by direct generalizations of arguments in [LU 2002] once the appropriate definitions are given. Hence, we will thoroughly describe the definitions and refer the reader to [LU 2002], noting any nontrivial changes. Throughout this section, we let $Q$ be an arbitrary smooth orbifold represented by the orbifold groupoid $\mathscr{G}$.

4A. The $M_{\Gamma}$-multiloop space of an orbifold. In this subsection, we develop a groupoid structure for a manifold $M_{\Gamma}$ with fundamental group $\Gamma$. This construction generalizes that of [LU 2002, Sections 3.1-3.2] for the case of $\Gamma=\mathbb{Z}$ and $M_{\Gamma}=S^{1}$.

Let $\Gamma$ be a finitely generated discrete group, let $M_{\Gamma}$ be a smooth manifold with fundamental group $\Gamma$, and let $M$ be the universal cover of $M_{\Gamma}$, so that $M / \Gamma=M_{\Gamma}$. We let $\pi_{\Gamma}: M \rightarrow M_{\Gamma}$ denote the covering projection. Fix a metric on $M_{\Gamma}$ and consider a cover $\mathscr{U}=\left\{U_{n}\right\}_{n \in \mathbb{N}}$ of $M_{\Gamma}$ that is $(1 / n)$-admissible; that is, each $U_{n}$ is evenly covered and has diameter $\leq 1 / n$. Note that if $M_{\Gamma}$ is compact, we can assume that $U$ is finite. Let $\mathcal{W}$ be the cover of $M$ formed by the connected components of the sets $\pi_{\Gamma}^{-1}\left(U_{i}\right)$ for each $U_{i} \in \mathcal{U}$. In other words, for each $n \in \mathbb{N}$, choose one

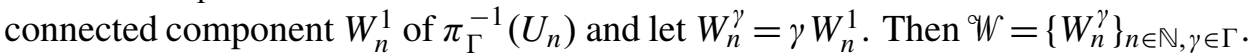
Set $W_{n}=\pi_{\Gamma}^{-1}\left(U_{n}\right)=\bigsqcup_{\gamma \in \Gamma} W_{n}^{\gamma}$, and define the groupoid $M^{\mathscr{W}}$ to be the groupoid associated to the covering $\mathscr{W}$ of $M$. That is, for $M^{\mathscr{W}}$ the set $\left(M^{\mathscr{W}}\right)_{0}$ of units and 
the set $\left(M^{\mathscr{W}}\right)_{1}$ of arrows are respectively given by

$$
\left(M^{\mathcal{W}}\right)_{0}=\coprod_{n \in \mathbb{N}, \gamma \in \Gamma} W_{n}^{\gamma} \quad \text { and } \quad\left(M^{\mathcal{W}}\right)_{1}=\coprod_{n, m \in \mathbb{N} ; \gamma, \delta \in \Gamma} W_{n}^{\gamma} \cap W_{m}^{\delta} .
$$

We let $\left(x, W_{n}^{\gamma}\right)$ denote the object associated to $x \in W_{n}^{\gamma} \subseteq M$ to distinguish it from $\left(x, W_{m}^{\delta}\right)$ in the case where $x \in W_{n}^{\gamma} \cap W_{m}^{\delta}$. When the specific translate of $W_{n}^{1}$ does not concern us, we simply use $\left(x, W_{n}\right)$. This introduces no ambiguity; $\Gamma$ is the group of deck translations of the manifold cover $M \rightarrow M_{\Gamma}$, so that $x$ can be contained in only one translate $W_{n}^{\gamma}$ of $W_{n}^{1}$. Similarly, we use $W_{n, m}^{\gamma, \delta}$ to denote the connected component $W_{n}^{\gamma} \cap W_{m}^{\delta}$ of $\left(M^{\mathcal{W}}\right)_{1}$ and let $W_{n, m}=\coprod_{\gamma, \delta \in \Gamma} W_{n, m}^{\gamma, \delta}$. Then $\left(x, W_{n, m}^{\gamma, \delta}\right)$ or simply $\left(x, W_{n, m}\right)$ (again, with no ambiguity) denotes the arrow corresponding to the point $x \in W_{n, m}^{\gamma, \delta}$. The structure maps are defined by

$$
\begin{array}{lr}
s_{M^{\mathscr{W}}}\left(x, W_{n, m}^{\gamma, \delta}\right)=\left(x, W_{n}^{\gamma}\right), & i_{M^{\mathscr{W}}}\left(x, W_{n, m}^{\gamma, \delta}\right)=\left(x, W_{m, n}^{\delta, \gamma}\right), \\
t_{M^{\mathscr{W}}}\left(x, W_{n, m}^{\gamma, \delta}\right)=\left(x, W_{m}^{\delta}\right), & u_{M^{\mathscr{W}}}\left(x, W_{n}^{\gamma}\right)=\left(x, W_{n, n}^{\gamma, \gamma}\right) ;
\end{array}
$$

a composable pair of arrows is of the form $\left(\left(x, W_{t, n}^{\nu, \gamma}\right),\left(x, W_{n, m}^{\gamma, \delta}\right)\right)$. We define the composition by $m_{M^{\mathscr{W}}}\left(\left(x, W_{t, n}^{v, \gamma}\right),\left(x, W_{n, m}^{\gamma, \delta}\right)\right)=\left(x, W_{t, m}^{v, \delta}\right)$.

Define a left $\Gamma$-action on $M^{\mathcal{W}}$ by

$$
\begin{aligned}
& \Gamma \times\left(M^{\mathscr{W}}\right)_{0} \rightarrow\left(M^{\mathscr{W}}\right)_{0}, \quad\left(\gamma^{\prime},\left(x, W_{n}^{\gamma}\right)\right) \mapsto\left(\gamma^{\prime} x, W_{n}^{\gamma^{\prime} \gamma}\right), \\
& \Gamma \times\left(M^{\mathscr{W}}\right)_{1} \rightarrow\left(M^{\mathscr{W}}\right)_{1}, \quad\left(\gamma^{\prime},\left(x, W_{n, m}^{\gamma, \delta}\right)\right) \mapsto\left(\gamma^{\prime} x, W_{n, m}^{\gamma^{\prime} \gamma, \gamma^{\prime} \delta}\right) .
\end{aligned}
$$

The following proposition is straightforward.

Proposition 4.1. The above is an action of the group $\Gamma$ on the Lie groupoid $M^{W}$.

Definition 4.2. Let $M_{\Gamma}^{\mathcal{W}}=\Gamma \ltimes M^{\mathscr{W}}$ be the groupoid crossed product of $\Gamma$ with the groupoid $M^{\text {W }}$ with respect to the above action of $\Gamma$. In particular, we have

$$
\begin{array}{ccc}
s_{M_{\Gamma}^{\mathscr{W}}}\left(\gamma,\left(x, W_{n, m}\right)\right)=\left(x, W_{n}\right), & i_{M_{\Gamma}^{\mathscr{W}}}\left(\gamma,\left(x, W_{n, m}\right)\right)=\left(\gamma^{-1},\left(\gamma x, W_{m, n}\right)\right), \\
t_{M_{\Gamma}^{\mathscr{W}}}\left(\gamma,\left(x, W_{n, m}\right)\right)=\left(\gamma x, W_{m}\right), & u_{M_{\Gamma}^{\mathscr{W}}}\left(x, W_{n}\right)=\left(1,\left(x, W_{n, n}\right)\right) ;
\end{array}
$$

a composable pair is of the form $\left(\gamma,\left(x, W_{l, n}\right)\right),\left(\delta,\left(\gamma x, W_{n, m}\right)\right)$, with composition given by

$$
m_{M_{\Gamma}^{\mathscr{W}}}\left[\left(\gamma,\left(x, W_{l, n}\right)\right),\left(\delta,\left(\gamma x, W_{n, m}\right)\right)\right]=\left(\gamma \delta,\left(x, W_{l, m}\right)\right) .
$$

Proposition 4.3. The groupoid $M_{\Gamma}^{\mathscr{W}}$ is Morita equivalent to $M_{\Gamma}$ with its trivial groupoid structure.

Proof. In fact, there is a strong equivalence from $M_{\Gamma}^{\mathscr{W}}$ to $M_{\Gamma}$ defined on objects by $\left(\gamma,\left(x, W_{n}\right)\right) \mapsto \pi_{\Gamma}(x)$, and on arrows by mapping $\left(\gamma,\left(x, W_{n, m}\right)\right)$ to the unit over $\pi_{\Gamma}(x)$. That this map is a strong equivalence is easy to check.

The next proposition is proved in the same way. 
Proposition 4.4. If $\tilde{\mathcal{W}}$ is a refinement of $\mathcal{W}$, then the natural groupoid morphism $\rho_{\tilde{W}}^{\tilde{W}}: M_{\Gamma}^{\tilde{W}} \rightarrow M_{\Gamma}^{\mathscr{W}}$ is a strong equivalence.

Proposition 4.3 implies that the Morita class of the groupoid $M_{\Gamma}^{\tilde{W}}$ is independent of the metric used to define it. Specifically, we may use Proposition 4.4 to see that if given two metrics on $M_{\Gamma}$ with corresponding covers $\bigcup_{1}$ and $\mho_{2}$ (inducing covers $W_{1}$ and $W_{2}$ of $\left.M\right)$, one can define a strictly smaller metric and corresponding cover $u_{3}$ that refines both $U_{1}$ and $U_{2}$.

Definition 4.5. Let $Q$ be a smooth orbifold presented by the orbifold groupoid $\mathscr{G}$, and let $\mathcal{W}$ be a cover of $M$ constructed from an admissible cover of $M_{\Gamma}$ as above. The $M_{\Gamma}$-multiloop groupoid of $\mathscr{G}$ corresponding to $\mathscr{W}$ is defined to be the groupoid $M \mathscr{L}(\mathscr{W} ; G)_{M_{\Gamma}}$, where

$$
\left(M \mathscr{L}(\mathscr{W} ; \mathscr{G})_{M_{\Gamma}}\right)_{0}=\operatorname{Hom}\left(M_{\Gamma}^{\mathscr{W}}, \mathscr{G}\right)
$$

is the set of Lie groupoid homomorphisms from $M_{\Gamma}^{\mathscr{W}}$ to $\mathscr{G}$. We define the arrows in $\mathcal{M L}(\mathcal{W} ; \mathscr{G})_{M_{\Gamma}}$ as follows. For any two elements $\Phi, \Psi \in \operatorname{Hom}\left(M_{\Gamma}^{\mathscr{W}}, \mathscr{G}\right)$, an arrow from $\Psi$ to $\Phi$ is a map $\Lambda:\left(M_{\Gamma}^{\mathscr{W}}\right)_{1} \rightarrow(\varphi)_{1}$ such that the diagram

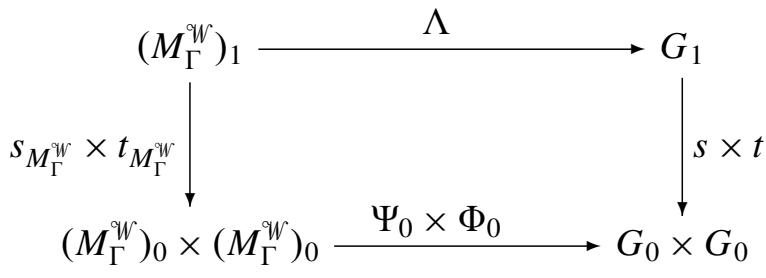

commutes and such that for every $\left(\gamma,\left(x, W_{n}\right)\right) \in\left(M_{\Gamma}^{\mathscr{W}}\right)_{1}$, we have

$$
\begin{aligned}
\Lambda\left(\gamma,\left(x, W_{n}\right)\right) & =\Psi_{1}\left(\gamma,\left(x, W_{n}\right)\right) \Lambda\left[u_{M_{\Gamma}^{\mathscr{W}} \circ s_{M_{\Gamma}^{\mathscr{W}}}}\left(\gamma,\left(x, W_{n}\right)\right)\right] \\
& =\Lambda\left[u_{M_{\Gamma}^{\mathscr{W}}} \circ t_{M_{\Gamma}^{\mathscr{W}}}\left(\gamma,\left(x, W_{n}\right)\right)\right] \Phi_{1}\left(\gamma,\left(x, W_{n}\right)\right),
\end{aligned}
$$

where as usual $\Phi_{1}$ and $\Psi_{1}$ denote the maps on arrows given by $\Phi$ and $\Psi$, respectively. The product above is taken in $G_{1}$, so that the target of the right element is equal to the source of the left.

If $\Lambda: \Psi \rightarrow \Phi$ and $\Omega: \Phi \rightarrow \Xi$, then the composition $\Omega \circ \Lambda$ is defined by

$$
\begin{aligned}
& \Omega \circ \Lambda\left[u_{M_{\Gamma}^{\mathscr{W}}} \circ t_{M_{\Gamma}^{\mathscr{W}}}\left(\gamma,\left(x, W_{n}\right)\right)\right] \\
& \quad=\Lambda\left[u_{M_{\Gamma}^{\mathscr{W}}} \circ t_{M_{\Gamma}^{\mathscr{W}}}\left(\gamma,\left(x, W_{n}\right)\right)\right] \Omega\left[u_{M_{\Gamma}^{\mathscr{W}}} \circ t_{M_{\Gamma}^{\mathscr{W}}}\left(\gamma,\left(x, W_{n}\right)\right)\right], \\
& \Omega \circ \Lambda\left(\gamma,\left(x, W_{n}\right)\right)=\Omega \circ \Lambda\left[u_{\left.M_{\Gamma}^{\mathscr{W}} \circ t_{M_{\Gamma}^{\mathscr{W}}}\left(\gamma,\left(x, W_{n}\right)\right)\right] \Xi_{1}\left(\gamma,\left(x, W_{n}\right)\right) .}\right.
\end{aligned}
$$

Under the compact-open topology, $\mathcal{M L}\left(\mathcal{W} ; \mathscr{G}_{M}\right.$ is a topological groupoid. For each arrow in $\Lambda \in M \mathscr{L}(\mathcal{W} ; \mathscr{G})_{M_{\Gamma}}$ from $\Phi$ to $\Psi, \Lambda \circ u_{M_{M_{\Gamma}}}$ is a natural transformation from $\Phi$ to $\Psi$ [Adem et al. 2007, Definition 1.40].

Compare the following to [LU 2002, Definition 3.2.2]. 
Definition 4.6. Let $Q$ be a smooth orbifold presented by the orbifold groupoid $\mathscr{G}$. The $M_{\Gamma}$-multiloop groupoid $\mathcal{M L}(\mathscr{G})_{M_{\Gamma}}$ of $\mathscr{G}$ is the colimit of the $\mathcal{M L}(\mathscr{W} ; \mathscr{G})_{M_{\Gamma}}$ over all admissible covers of $M_{\Gamma}$ partially ordered by inclusion of cover charts.

Lemma 4.7. Let $M \mathscr{L}(\mathscr{G})_{M_{\Gamma}}$ be the $M_{\Gamma}$-multiloop groupoid of an orbifold $Q$ presented by the orbifold groupoid $G$. Then any arrow $\Lambda: \Psi \rightarrow \Phi$ is completely determined by $\Psi$ and by $\Lambda \circ u_{M_{\Gamma}^{\mathscr{W}}}\left(x, W_{n}\right)$ for any $\left(x, W_{n}\right) \in\left(M_{\Gamma}^{\mathscr{W}}\right)_{0}$.

Proof. It is straightforward from the definitions [LU 2002, Lemma 3.2.4]. On any given $W_{n}, \Lambda$ is determined by $\Psi$, by a single value $\Lambda \circ u_{M_{\Gamma}^{\Psi}}\left(x, W_{n}\right)$, and by (4-1). This determines $\Lambda$ on each chart $W_{m}$ such that $W_{n} \cap W_{m} \neq \varnothing$, and hence recursively on every chart.

Proposition 4.8. Let $\mathcal{M L}(\mathscr{G})_{M_{\Gamma}}$ be the $M_{\Gamma}$-multiloop groupoid of an orbifold $Q$ presented by the orbifold groupoid $\mathscr{G}$. Then $\mathcal{M L}(\mathscr{G})_{M_{\Gamma}}$ is étale.

Proof. The proof is straightforward from the definitions. An arrow $\Lambda: \Psi \rightarrow \Phi$ is determined by $\Psi$ and a single value $\Lambda \circ u_{M_{\Gamma}^{W}}\left(x, W_{n}\right)$. The result then follows from the fact that the isotropy groups of $\mathscr{G}$ are finite.

Proposition 4.9. Let $\mathcal{M L}\left(\mathscr{G}_{i}\right)_{M_{\Gamma}}$ be the $M_{\Gamma}$-multiloop groupoid of orbifolds $Q_{i}$ presented by the orbifold groupoids $\varphi_{i}$ for $i=1,2$. A groupoid homomorphism $e: \mathscr{G}_{1} \rightarrow \mathscr{G}_{2}$ induces a homomorphism

$$
e_{\mathcal{M L}}: \mathcal{M L}\left(\mathscr{G}_{1}\right)_{M_{\Gamma}} \rightarrow \mathcal{M L}\left(\mathscr{G}_{2}\right)_{M_{\Gamma}}
$$

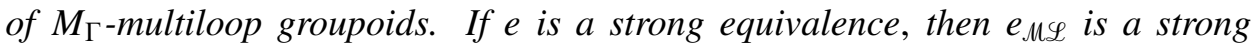
equivalence.

Proof. See the proof of [LU 2002, Definition 3.4.1 and Lemma 3.4.2].

4B. The $M_{\Gamma}$-multiloops when $\Gamma$ is a subgroup of a contractible abelian group. In this section, we assume that $\Gamma$ is a subgroup of a contractible abelian Lie group $T$; in the notation of Section $4 \mathrm{~A}, T=M$ and $T / \Gamma=M_{\Gamma}$. Following [LU 2002, Section 3.6], we recover the $\Gamma$-sectors of $Q$ from the fixed points of the $T / \Gamma$-multiloop groupoid. First, we define a $T$-action on the $T / \Gamma$-multiloop groupoid $M \mathscr{L}(\mathscr{G})_{T / \Gamma}$.

Definition 4.10. Suppose $\Gamma$ is a subgroup of a contractible abelian Lie group $T$. Let $\mathcal{L} \mathscr{L}(\mathcal{W} ; \mathscr{G})_{T / \Gamma}$ be the $T / \Gamma$-multiloop groupoid associated to the cover $\mathcal{W}$ of $T$ given by Definition 4.5. For each $W_{n}=\bigsqcup_{\gamma \in \Gamma} W_{n}^{\gamma}$ and $t \in T$, let $W_{n}^{t}$ denote the translate $t W_{n}=\left\{t x: x \in W_{n}\right\}$, and let $\mathcal{W}^{t}$ denote the translated cover $\left\{W_{n}^{t}\right\}_{n \in \mathbb{N}}$. This introduces no ambiguity; $W_{n}^{t}$ has the same meaning as in Section 4A when $t \in \Gamma \leq T$. Then $T$ acts on $\coprod_{t \in T} \mathscr{W}^{t}$ via $\left(s,\left(x, W_{n}^{t}\right)\right) \mapsto\left(s x, W_{n}^{s t}\right)$ for $s \in T$. Since $T$ is abelian, this action descends to a $T$-action on the cover $\coprod_{n \in \mathbb{N}, t \Gamma \in T / \Gamma} U_{n}^{t \Gamma}$ of $T / \Gamma$-translates of $U$ in the same way. 
Now define an action of $T$ on $\bigsqcup_{t \in T} \mathcal{M L}\left(\mathscr{W}^{t} ; \mathscr{G}\right)_{T / \Gamma}$ by

$$
T \times\left(\coprod_{t \in T} \mathcal{M L}\left(\mathscr{W}^{t} ; \mathscr{G}\right)_{T / \Gamma}\right)_{0} \rightarrow\left(\coprod_{t \in T} \mathcal{M L}\left(\mathscr{W}^{t} ; \mathscr{G}\right)_{T / \Gamma}\right)_{0}, \quad(t, \Psi) \mapsto \Psi^{t},
$$

where $\Psi^{t}$ is defined by

$$
\Psi_{0}^{t}\left(x, W_{n}^{t}\right)=\Psi\left(t^{-1} x, W_{n}\right) \quad \text { and } \quad \Psi_{1}^{t}\left(\gamma,\left(x, W_{n, m}^{t}\right)\right)=\Psi_{1}\left(\gamma,\left(t^{-1} x, W_{n, m}\right)\right) .
$$

Taking the colimit, we obtain an action of $T$ on $\mathcal{M L}(\mathscr{G})_{T / \Gamma}$.

Now consider the subgroupoid $\mathcal{M L}(\mathscr{G})_{T / \Gamma}^{T}$ of $\mathcal{M L}(\mathscr{G})_{T / \Gamma}$ consisting of elements fixed by the action of $T$. In Theorem 4.13, we will show that $\mathcal{M L}(\mathscr{G})_{T / \Gamma}^{T}$ is Morita equivalent to $\varphi \Gamma$, the groupoid presenting the $\Gamma$-sectors of $Q$. This coincides with [LU 2002, Theorem 3.6.4 and Proposition 3.6.6] when $\Gamma=\mathbb{Z}$; see also [de Fernex et al. 2006]. The next two lemmas can be proved in the same way as in [LU 2002, Lemmas 3.6.2 and 3.6.3]. We give the proof of Lemma 4.12 explicitly, as it is important for the proof of Theorem 4.13.

Lemma 4.11. For any object $\Psi$ of $\mathcal{M L}(\mathscr{G})_{T / \Gamma}^{T}, \Psi_{0}$ and $\Psi_{1}$ are locally constant.

Lemma 4.12. For any object $\Psi$ of $M \mathscr{L}(\mathscr{G})_{T / \Gamma}^{T}$, there exists another object $\Phi$ of $M \mathscr{L}(\mathscr{G})_{T / \Gamma}^{T}$ defined over the trivial cover of $T$ by one chart such that there is an arrow $\Lambda$ connecting $\Psi$ and $\Phi$.

Proof. Given a point $(x, W) \in\left(T^{\mathscr{W}}\right)_{0}$, if $\left(y, W^{\prime}\right) \in\left(T^{\mathscr{W}}\right)_{0}$ is another point in $\left(T^{\mathscr{W}}\right)_{0}$, then there exists a finite collection $W^{1}, \ldots, W^{r}$ of sets in the cover $W$ such that $W^{1}=W, W^{r}=W^{\prime}$, and $W^{i} \cap W^{i+1} \neq \varnothing$ in $T$ for $i=1,2, \ldots, r-1$.

For each $\left(z_{i}, W^{i}\right)$ such that $z_{i} \in W^{i} \cap W^{i+1}$, we have that $\Psi_{1}\left(1,\left(z_{i}, W^{i} \cap W^{i+1}\right)\right)$ is an arrow from $\Psi_{0}\left(z_{i}, W^{i}\right)$ to $\Psi_{0}\left(z_{i}, W^{i+1}\right)$. Define the arrow $A_{i}^{i+1}\left(z_{i}\right) \in\left(T^{\mathscr{W}}\right)_{1}$ from $\Psi_{0}\left(z_{i}, W^{i}\right)$ to $\Psi_{0}\left(z_{i}, W^{i+1}\right)$ by $A_{i}^{i+1}\left(z_{i}\right)=\Psi_{1}\left(1,\left(z_{i}, W^{i} \cap W^{i+1}\right)\right)$, and note that since $\Psi_{0}$ and $\Psi_{1}$ are locally constant by Lemma 4.11, $A_{i}^{i+1}$ does not depend on $z_{i}$. Choosing one $z_{i} \in W^{i} \cap W^{i+1}$ for each $i$, we set

$$
\begin{aligned}
A_{W}^{W^{\prime}}= & \Psi_{1}\left(1,\left(z_{r-1}, W^{r-1} \cap W^{r}\right)\right) \Psi_{1}\left(1,\left(z_{r-2}, W^{r-2} \cap W^{r-1}\right)\right) \\
& \ldots \Psi_{1}\left(1,\left(z_{1}, W^{1} \cap W^{2}\right)\right) .
\end{aligned}
$$

The definition of $A_{W}^{W^{\prime}}$ depends only on the sets $W$ and $W^{\prime}$, and that $\left(A_{W}^{W^{\prime}}\right)^{-1}=A_{W^{\prime}}^{W}$. Define a morphism $\Phi: T_{\Gamma}^{\mathscr{W}} \rightarrow \mathscr{G}$ by

$$
\Phi_{0}\left(z, W_{n}\right)=\Psi_{0}(x, W) \quad \text { and } \quad \Phi_{1}\left(\gamma,\left(z, W_{n, m}\right)\right)=A_{W_{n}}^{W_{m}} \Psi_{1}\left(\gamma,\left(z, W_{m, n}\right)\right) A_{W_{n}}^{W_{m}} .
$$

Because $\Psi_{1}$ is locally constant, we have for each $z \in W_{n, m}$ that

$$
\begin{aligned}
& \Phi_{1}\left(1,\left(z, W_{n, m}\right)\right)=\Psi_{1}\left(1,\left(z, W_{n, m}\right)\right), \\
& \Phi_{1}\left(\gamma,\left(z, W_{n, m}\right)\right)=\Psi_{1}\left(\gamma,\left(z, W_{n, m}\right)\right) .
\end{aligned}
$$


Hence we can define $\Phi$ on the trivial cover of $T$ consisting of points $(z, T)$ by

$$
\Phi_{0}(z, T)=\Phi_{0}(x, W) \quad \text { and } \quad \Phi_{1}(\gamma,(z, T))=\Phi_{1}\left(\gamma,\left(z, W_{n, m}\right)\right),
$$

whenever $z \in W_{n} \cap W_{m}$. Lastly, we define an arrow $\Lambda: \Psi \rightarrow \Phi$ by

$$
\Lambda\left(1,\left(z, W_{n, m}\right)\right)=A_{W_{n}}^{W_{m}} .
$$

Theorem 4.13. There is a strong equivalence from $\mathcal{M L}(\varphi)_{T / \Gamma}^{T}$ to the groupoid $\varphi^{\Gamma}$ of $\Gamma$-sectors of $Q$.

Proof. See the proof of [LU 2002, Theorem 3.6.4]. Given $\Psi \in \mathcal{M} \mathscr{L}(\mathscr{G})_{T / \Gamma}^{T}$, let $\Phi$ be as in the proof of Lemma 4.12. Since $\Phi_{0}$ is locally constant, $\Phi_{0}(y, T)=\Phi_{0}(1, T)$ for each $y \in T$. We have

$$
s \circ \Phi_{1}(\gamma,(y, T))=\Phi_{0}(y, T)=\Phi_{0}(\gamma y, T)=t \circ \Phi_{1}(\gamma,(y, T)),
$$

so that each $\Phi_{1}(\gamma,(y, T))$ is an element of the isotropy group $G_{\Phi_{0}(y)}=G_{\Phi_{0}(1)}$. Hence, we can define a homomorphism $\phi: \Gamma \rightarrow G_{\Phi_{0}(1)}$ by $\phi(\gamma)=\Phi_{1}(\gamma,(1, T))$. Clearly, the correspondence $\Psi \mapsto \phi$ is surjective, since given any $\phi_{x}: \Gamma \rightarrow G_{x}$, one can define a $\Psi \in \mathcal{M L}(\mathscr{G})_{T / \Gamma}^{T}$ with $\Psi_{0}(y, T)=x$ and $\Psi_{1}(\gamma,(y, T))=\phi_{x}(\gamma)$. That this correspondence is a strong equivalence of groupoids is straightforward to demonstrate.

It follows that $\mathcal{M L}(\mathscr{G})_{T / \Gamma}^{T}$ is Morita equivalent to $\varphi{ }^{\Gamma}$.

Chen [2006, Proposition 3.5.3] proved that in the case of a proper étale topological groupoid $\mathscr{G}$ representing an orbispace such that the space of objects is a $T_{1}$ space, there is an identification similar to that given by Theorem 4.13 on the level of orbispaces for the case $\Gamma=\mathbb{Z}$. Using exactly the same proof with the definitions given above and Equation (2-1), we have the following.

Proposition 4.14. Let $X$ be an étale proper orbispace, that is, an orbispace represented by the étale proper groupoid $G$, such that $G_{0}$ is a $T_{1}$ space. Let $\Gamma$ be a discrete subgroup of a contractible abelian Lie group $T$. Then the orbit space $\left|\mathcal{M L}(\mathscr{G})_{T / \Gamma}^{T}\right|$ of the groupoid $\mathcal{M L}(\mathscr{G})_{T / \Gamma}^{T}$ is homeomorphic to $\left|\mathscr{G}^{\Gamma}\right|$.

4C. The $M_{\Gamma}$-multiloop and the $\Gamma$-sectors in the general case. In the general case of $M_{\Gamma}$ an arbitrary manifold with fundamental group $\Gamma$ and universal cover $\Gamma$, we have a correspondence similar to Theorem 4.13. In this case, we use the groupoid of constants, a subgroupoid of $\mathcal{M L}(\mathscr{G})_{M_{\Gamma}}$.

Definition 4.15. Let $Q$ be an orbifold presented by the orbifold groupoid $\mathscr{G}$. The groupoid of constants $\mathscr{C}(\mathscr{G})_{M_{\Gamma}}$ of $\mathcal{M L}(\mathscr{G})_{M_{\Gamma}}$ is defined to be the subgroupoid of $\mathcal{M L}(\mathscr{G})_{M_{\Gamma}}$ consisting of the $\Phi$ such that $\sigma \circ \Phi$ is constant. Recall that $\sigma: \mathscr{G} \rightarrow|\mathscr{G}|$ denotes the quotient map onto the orbit space of $\mathscr{G}$. 
Theorem 4.16. There is a strong equivalence between the groupoid of constants $\mathscr{C}(\mathscr{G})_{M_{\Gamma}}$ and the groupoid $\varphi{ }^{\Gamma}$ of $\Gamma$-sectors of $Q$.

The proof is identical to that of Theorem 4.13.

4D. The $M_{\Gamma}$-multiloops of a quotient orbifold. In this section, we specialize to the case where $M_{\Gamma}$ is compact and $Q$ is presented as the quotient of a smooth connected manifold $X$ by a compact Lie group $G$ acting locally freely (that is, properly with discrete stabilizers). In the case of $G$ finite, a very explicit characterization of the loop space is given in [LU 2002, Section 4.1], where it was shown that it is enough to consider only the homomorphisms defined on the trivial cover. Here, we briefly explain how this characterization extends readily to the case of $G$ compact and the $M_{\Gamma^{-}}$-multiloops. Throughout this section, we let $\mathscr{G}$ denote an orbifold groupoid Morita equivalent to $G \ltimes X$. In particular, we can take $\mathscr{G}$ to be given by a collection of slices for the $G$-action as in Theorem 3.5.

The proof of the following is similar to that of Lemma 4.12 and, given the modifications outlined in Definitions 4.2, 4.5, and 4.15 and the local structure of quotient orbifolds demonstrated by Theorem 3.5, identical to that of [LU 2002, Lemma 4.1.1].

Proposition 4.17. Let $Q$ be a quotient orbifold presented by $G \ltimes X$ with $G$ a compact Lie group acting locally freely on the smooth manifold $X$. Let $M \mathscr{L}(\mathscr{G})_{M_{\Gamma}}$ be the $M_{\Gamma}$-multiloops for $M_{\Gamma}$ compact. Then for any morphism $\Psi: M_{\Gamma}^{\mathscr{W}} \rightarrow \mathscr{G}$, there

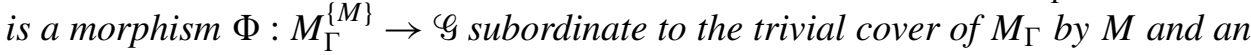
arrow connecting $\Psi$ to $\Phi$.

Similarly, a morphism $\Psi$ subordinated to the trivial cover of $M_{\Gamma}$ is determined by the image of $\coprod_{i=1}^{s}\left\{\gamma_{i}\right\} \times M$ under $\Psi_{1}$ where $\left\{\gamma_{1}, \ldots, \gamma_{s}\right\}$ is a set of generators of $\Gamma$; compare [LU 2002, Section 3.3].

Lemma 4.18. Let $Q$ be a quotient orbifold presented by $G \ltimes X$ with $G$ a compact Lie group acting locally freely on the smooth manifold $X$. Every morphism in $\Psi \in \mathcal{M L}(\mathscr{G})_{M_{\Gamma}}$ subordinated to the trivial cover of $M_{\Gamma}$ is determined by the image of $\coprod_{i=1}^{s}\left\{\gamma_{i}\right\} \times M$ under $\Psi_{1}$, where $\left\{\gamma_{1}, \ldots, \gamma_{s}\right\}$ is a set of generators of $\Gamma$.

Proof. Pick a set of generators $\left\{\gamma_{1}, \ldots, \gamma_{s}\right\}$ of $\Gamma$ and let $(\gamma, x) \in \Gamma \times M$. Then if $\gamma=\gamma_{\alpha_{1}}^{\beta_{1}} \ldots \gamma_{\alpha_{n}}^{\beta_{n}}$ is an expression of $\gamma$ in terms of these generators, we have

$$
(\gamma, x)=\left(\gamma_{\alpha_{1}}, \gamma_{\alpha_{1}}^{\beta_{1}-1} \cdots \gamma_{\alpha_{n}}^{\beta_{n}} x\right) \cdots\left(\gamma_{\alpha_{n}}, \gamma_{\alpha_{n}} x\right)\left(\gamma_{\alpha_{n}}, x\right) .
$$

It follows that

$$
\Psi_{1}(\gamma, x)=\Psi_{1}\left(\gamma_{\alpha_{1}}, \gamma_{\alpha_{1}}^{\beta_{1}-1} \cdots \gamma_{\alpha_{n}}^{\beta_{n}} x\right) \cdots \Psi_{1}\left(\gamma_{\alpha_{n}}, \gamma_{\alpha_{n}} x\right) \Psi_{1}\left(\gamma_{\alpha_{n}}, x\right) .
$$

Fixing a generating set $\left\{\gamma_{1}, \ldots, \gamma_{s}\right\}$ of $\Gamma$, it follows that there is a bijective correspondence between the morphisms $\Psi$ subordinated to the trivial cover of $M_{\Gamma}$ 
in $\mathcal{M L}(\mathscr{G})_{\Gamma}$ and the set of pairs $(f, \Theta)$, where $\Theta=\left\{g_{1}, \ldots, g_{s}\right\}$ is an $s$-tuple of elements of $G$ satisfying the same relations as the $\gamma_{i}$, and $f: M \rightarrow X$ is a smooth map such that $g_{i} f(x)=f\left(\gamma_{i} x\right)$ for each $i=1, \ldots s$. Let $\mathscr{P}_{\Theta}$ denote the set of all such pairs. Similarly, let $\Lambda$ be an arrow between homomorphisms $\Psi=(f, \Theta)$ and $\Phi=\left(f^{\prime}, \Upsilon\right)$ with $\Theta=\left\{g_{1}, \ldots, g_{s}\right\}$ and $\Upsilon=\left\{k_{1}, \ldots, k_{s}\right\}$. Because $X$ is connected, there is an $h \in G$ such that $k_{i}=h g_{i} h^{-1}$ and $h f(x)=f^{\prime}(x)$ for each $i=1, \ldots s$ and $x \in M$. Then the following is a consequence of Lemma 4.7.

Proposition 4.19. Let $G$ act on $\mathscr{P}_{\Theta}$ via

$$
[h,(f, \Theta)] \mapsto\left(h f, h \Theta h^{-1}\right),
$$

where $h \Theta h^{-1}$ indicates pointwise conjugation of the s-tuple $\Theta$. The crossed product groupoid $G \ltimes \mathscr{P}_{\Theta}$ is Morita equivalent to the orbifold $M_{\Gamma}-$ multiloop groupoid $\mathcal{M L}(\mathscr{G})_{M^{\Gamma}}$ of $Q$.

For each $s$-tuple $\Theta=\left(g_{1}, \ldots, g_{s}\right)$, let $C_{G}(\Theta)$ denote the centralizer of the subgroup generated by the $g_{i}$. Techniques identical to those in Lemma 2.3 demonstrate that the crossed-product groupoid $G \ltimes \mathscr{P}_{\Theta}$ is Morita equivalent to

$$
\coprod\left(C_{G}(\Theta) \ltimes \mathscr{P}_{\Theta}\right),
$$

where the sum is over the $G$-conjugacy classes $(\Theta)$ of the $s$-tuples $\Theta$. Therefore:

Corollary 4.20. The groupoid $\coprod_{(\Theta)}\left(C_{G}(\Theta) \ltimes \mathscr{P}_{\Theta}\right)$ is Morita equivalent to the orbifold $M_{\Gamma}$-multiloop groupoid $\mathcal{M L}(\mathscr{G})_{M^{\Gamma}}$ of $Q$.

In [Tamanoi 2003, Equation 2-12, page 808], the multiloop $\Gamma$-sectors for global quotients are given by

$$
\mathbb{L}_{M_{\Gamma}}(X ; G)=\coprod_{(\phi)} \operatorname{Map}_{\phi}(M ; X) / C_{G}(\phi),
$$

where the space $\operatorname{Map}_{\phi}$ is defined as

$$
\operatorname{Map}_{\phi}(M ; X)=\{f: M \rightarrow X \mid f(\gamma x)=\phi(\gamma) f(x) \text { for all } x \in M, \gamma \in \Gamma\}
$$

(with notation modified to our case, and the $\Gamma$-action on $M$ expressed as a left action). Hence $\mathbb{L}_{M_{\Gamma}}$ coincides with the groupoid in Corollary 4.20 in the case of $G$ finite.

Restricting to the groupoid of constant maps $(f, \Theta)$ in $M L(G)_{M_{\Gamma}}$ as we did in Definition 4.15, we have that if $\Theta=\left(g_{1}, \ldots, g_{s}\right)$, then

$$
g_{i} f(x)=f\left(\gamma_{i} x\right)=f(x) \text { for each } x \in X \text { and } i=1, \ldots, s .
$$

Hence the image of $f$ is fixed by each $g_{i}$. 
Corollary 4.21. The subgroupoid of constants $\mathscr{C} L(\mathscr{G})_{M_{\Gamma}}$ of $\mathcal{M}(G)_{\Gamma}$ is given by

$$
\coprod\left(C_{G}(\Theta) \ltimes X^{\langle\Theta\rangle}\right),
$$

$(\Theta)$

where the sum is over the $G$-conjugacy classes $(\Theta)$ of the s-tuples $\Theta$, and is Morita equivalent to the groupoid $\varphi^{\Gamma}$ presenting the $\Gamma$-sectors $\tilde{Q}_{\Gamma}$.

\section{Acknowledgment}

Farsi thanks the Mathematical Sciences Research Institute for its hospitality during the preparation of this paper.

\section{References}

[Adem and Ruan 2003] A. Adem and Y. Ruan, "Twisted orbifold $K$-theory", Comm. Math. Phys. 237:3 (2003), 533-556. MR 2004e:19004 Zbl 1051.57022

[Adem et al. 2007] A. Adem, J. Leida, and Y. Ruan, Orbifolds and stringy topology, Cambridge Tracts in Mathematics 171, Cambridge University Press, 2007. MR 2009a:57044 Zbl 1157.57001

[Boileau et al. 2003] M. Boileau, S. Maillot, and J. Porti, Three-dimensional orbifolds and their geometric structures, Panoramas et Synthèses 15, Société Mathématique de France, Paris, 2003. MR 2005b:57030 Zbl 1058.57009

[Bryan and Fulman 1998] J. Bryan and J. Fulman, "Orbifold Euler characteristics and the number of commuting $m$-tuples in the symmetric groups", Ann. Comb. 2:1 (1998), 1-6. MR 2000f:20002 Zbl 0921.55003

[Chen 2006] W. Chen, "On a notion of maps between orbifolds, II: Homotopy and CW-complex", Commun. Contemp. Math. 8:6 (2006), 763-821. MR 2007g:57045 Zbl 1108.22002

[Chen and Ruan 2004] W. Chen and Y. Ruan, "A new cohomology theory of orbifold", Comm. Math. Phys. 248:1 (2004), 1-31. MR 2005j:57036 Zbl 1063.53091

[Crainic and Moerdijk 2001] M. Crainic and I. Moerdijk, "Foliation groupoids and their cyclic homology", Adv. Math. 157:2 (2001), 177-197. MR 2002a:22004 Zbl 0989.22010

[tom Dieck 1987] T. tom Dieck, Transformation groups, de Gruyter Studies in Mathematics 8, de Gruyter, Berlin, 1987. MR 89c:57048 Zbl 0611.57002

[de Fernex et al. 2006] T. de Fernex, E. Lupercio, T. Nevins, and B. Uribe, "A localization principle for orbifold theories", pp. 113-133 in Recent developments in algebraic topology, edited by A. Adem et al., Contemp. Math. 407, Amer. Math. Soc., Providence, RI, 2006. MR 2007e:58030 Zbl 1126.58012

[FS 2010] C. Farsi and C. Seaton, "Nonvanishing vector fields on orbifolds", Trans. Amer. Math. Soc. 362:1 (2010), 509-535. MR 2550162

[Henriques and Gepner 2007] A. Henriques and D. Gepner, "Homotopy theory of orbispaces", preprint, 2007. arXiv math/0701916

[Henriques and Metzler 2004] A. Henriques and D. S. Metzler, "Presentations of noneffective orbifolds", Trans. Amer. Math. Soc. 356:6 (2004), 2481-2499. MR 2005a:58027 Zbl 1060.58013

[Kawasaki 1978] T. Kawasaki, “The signature theorem for $V$-manifolds”, Topology 17:1 (1978), 75-83. MR 57 \#14072 Zbl 0392.58009 
[Kawasaki 1979] T. Kawasaki, “The Riemann-Roch theorem for complex $V$-manifolds", Osaka J. Math. 16:1 (1979), 151-159. MR 80f:58042 Zbl 0405.32010

[Kawasaki 1981] T. Kawasaki, "The index of elliptic operators over $V$-manifolds", Nagoya Math. J. 84 (1981), 135-157. MR 83i:58095 Zbl 0437.58020

[Leida 2005] J. Leida, “Orbifolds and stable homotopy groups”, preprint, 2005. arXiv math/0505431

[LU 2002] E. Lupercio and B. Uribe, "Loop groupoids, gerbes, and twisted sectors on orbifolds", pp. 163-184 in Orbifolds in mathematics and physics (Madison, WI, 2001), edited by A. Adem et al., Contemp. Math. 310, Amer. Math. Soc., Providence, RI, 2002. MR 2004c:58043 Zbl 1041.58008

[Moerdijk 2002] I. Moerdijk, "Orbifolds as groupoids: An introduction”, pp. 205-222 in Orbifolds in mathematics and physics (Madison, WI, 2001), edited by A. Adem et al., Contemp. Math. 310, Amer. Math. Soc., Providence, RI, 2002. MR 2004c:22003 Zbl 1041.58009

[Moerdijk and Mrčun 2003] I. Moerdijk and J. Mrčun, Introduction to foliations and Lie groupoids, Cambridge Studies in Advanced Mathematics 91, Cambridge University Press, 2003. MR 2005c: 58039 Zbl 1029.58012

[Ruan 2002] Y. Ruan, "Stringy geometry and topology of orbifolds", pp. 187-233 in Symposium in Honor of C. H. Clemens (Salt Lake City, UT, 2000), edited by A. Bertram et al., Contemp. Math. 312, Amer. Math. Soc., Providence, RI, 2002. MR 2004b:32051 Zbl 1060.14080

[Tamanoi 2001] H. Tamanoi, "Generalized orbifold Euler characteristic of symmetric products and equivariant Morava K-theory”, Algebr. Geom. Topology 1 (2001), 115-141. MR 2002e:57052 Zbl 0965.57033

[Tamanoi 2003] H. Tamanoi, "Generalized orbifold Euler characteristics of symmetric orbifolds and covering spaces”, Algebr. Geom. Topology 3 (2003), 791-856. MR 2005j:57025 Zbl 1037.57022

[Thurston 1978] W. Thurston, "The geometry and topology of 3-manifolds", lecture notes, Princeton University Math Dept., 1978, available at http://www.msri.org/publications/books/gt3m.

Received March 6, 2009.

CARLA FARSi

DEPARTMENT OF MATHEMATICS

UNIVERSITY OF COLORADO AT BOULDER

CAMPUS BOX 395

BOULDER, CO 80309-0395

UNITED STATES

farsi@euclid.colorado.edu

http://www.colorado.edu/math/people/professors/farsi.html

Christopher SEATON

DEPARTMENT OF MATHEMATICS AND COMPUTER SCIENCE

RHODES COLlEgE

2000 NORTH PARKWAY

MEMPHIS, TN 38112-1690

UNITED STATES

seatonc@rhodes.edu

http://faculty.rhodes.edu/seaton/ 